NISSUNA UMANA INVESTIGAZIONE SI PUO DIMANDARE VERA SCIENZIA S'ESSA NON PASSA PER LE MATEMATICHE DIMOSTRAZIONI LEONARDO DA VINCI

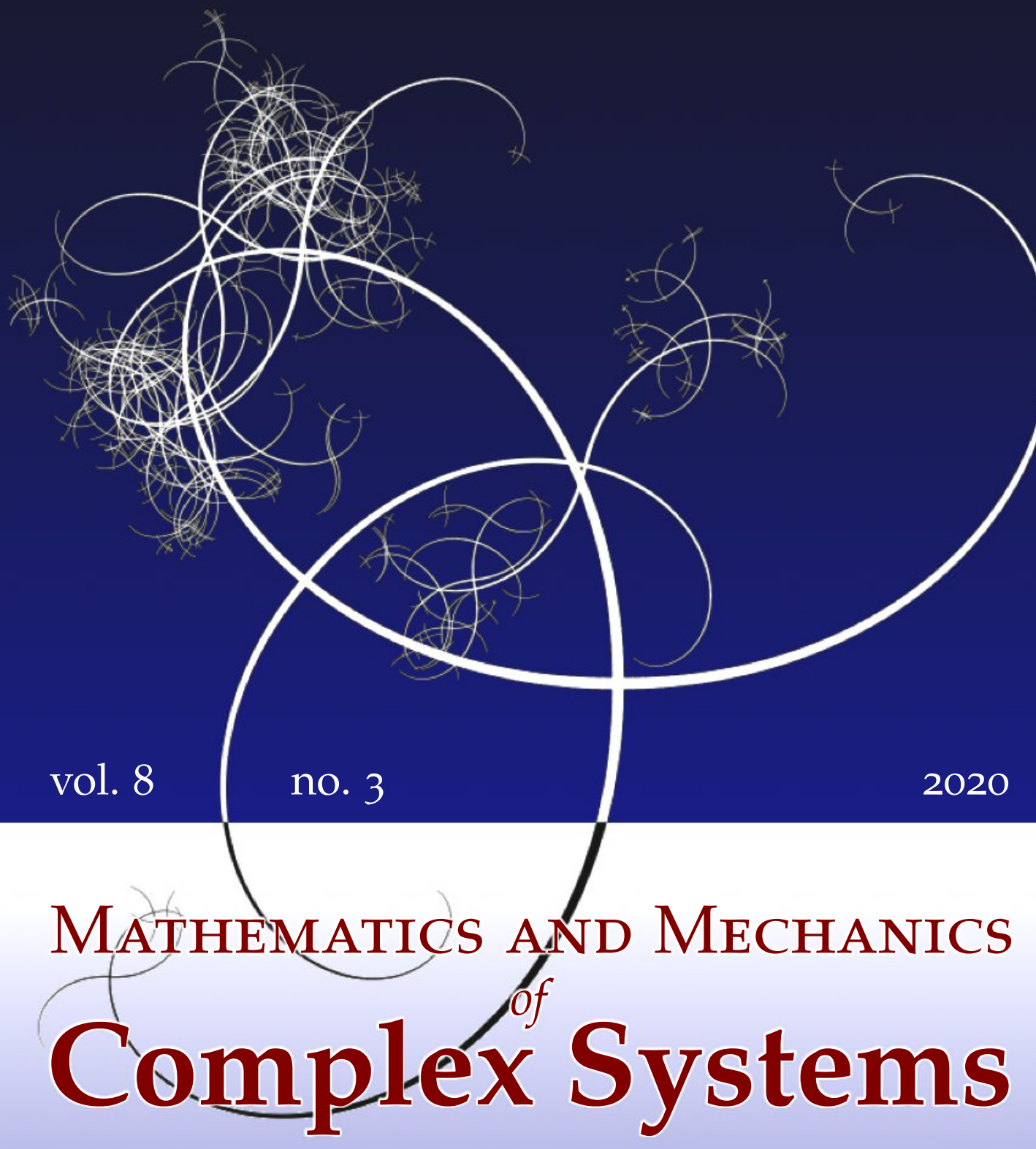

GianPietro Del Piero

ON CLASSICAL CONTINUUM MECHANICS, TWO-SCALE CONTINUA, AND PLASTICITY 


\title{
ON CLASSICAL CONTINUUM MECHANICS, TWO-SCALE CONTINUA, AND PLASTICITY
}

\author{
GianPietro Del Piero
}

\begin{abstract}
The paper starts with a careful analysis of the kinematics of two-scale continua. The subsequent developments are based on a single additional concept, the concept of energy. Two basic axioms are formulated in energetic terms, and the stress tensors, the constitutive equations, and all other elements required for the formulation of the initial/boundary-value problem are regarded as derived quantities. A comparison with the theory of gradient plasticity shows the innovative aspects of the proposed theory.
\end{abstract}

\section{Introduction}

This paper is a revisitation of some ideas developed by the present author in a series of papers [Del Piero 2009; 2014a; 2014b; 2017; 2018a; 2018b; 2019] concerning the principles of classical mechanics, their extension to generalized continua, and their application to plasticity. Over the years, many ideas have evolved and some became obsolete. Here I make an attempt to fix the present state of the art. I chose to restrict my exposition to two-scale continua because, being a first step toward a generalization of classical mechanics, such continua must be well understood before proceeding to further steps.

For the theoretical bases of classical mechanics and for the notation, I follow [Truesdell and Noll 1965; Noll 1973; Truesdell 1991; Gurtin 1981]. For generalized continua, I refer to the book [Gurtin et al. 2010]. I also frequently make reference to my previous paper [Del Piero 2019]. In particular, the following Section 2 on the two-scale geometric representation of deformable bodies is a summary of Chapters 2-4 of [Del Piero 2019], to which the interested reader is referred for further details.

Most treatises on mechanics, including those cited above, can be subdivided into three parts: kinematics, forces or interactions, and constitutive equations. In the passage from the first to the second part, the introduction of objects called forces

\section{Communicated by Miroslav Šilhavý.}

MSC2010: primary 74AXX, 74A05; secondary 74A60, 74C15, 74C20.

Keywords: foundations of mechanics, two-scale continua, reference frames, gradient plasticity, nonlocal flow rules. 
involves an abrupt change of language and rigor. Since their systematic introduction by Newton, to many authors forces appeared as mysterious objects, and the belief in their existence was frequently considered as an act of faith. ${ }^{1}$ Regarding "rigor", I reproduce three quotes taken from [Gurtin et al. 2010, §19]:

- "[forces are] obscure and metaphysical beings, capable of nothing but spreading darkness over a science clear by itself" (d'Alembert),

- "we speak of forces only to conceal our ignorance" (Maupertuis),

- "[forces are] an obscure metaphysical notion" (Carnot).

Even Mach's definition ${ }^{2}$

- "force is any circumstance of which the consequence is motion"

does not seem to be illuminating. The main argument in favor of the introduction of forces seems to be that they correctly describe the observed phenomena, which is more or less the same argument of the supporters of the Ptolemaic versus the Copernican cosmology.

For a long time I was puzzled by this difference in the presentations of kinematics and of forces/interactions. Slowly, I started to realize that the difference disappears if energy were taken as a primitive concept. When doing so, the only mathematical object to be added to those of kinematics is a functional involving the deformation measures, local or nonlocal in space, and possibly their past time histories. In this way, the mysterious objects become derived quantities of the energy.

There are historical reasons why this simple and rational approach was not adopted ab initio. Traditionally, energy was conceived as reversible, so that an energetic approach was considered appropriate only to conservative systems. For the study of irreversible processes, a thermodynamic approach was adopted instead. The energy was subdivided into a recoverable part, the free energy, and a part proportional to the entropy of the body. In this way the thermal variables, which by their own nature are extraneous to continuum mechanics, became an essential part of the picture. This was mainly due to the ignorance of the concept of dissipation potential elaborated by the French school, ${ }^{3}$ which renders possible a purely mechanical energetic approach to dissipative systems. ${ }^{4}$

Section 3 is devoted to the external energy, that is, to the energy supplied to, or extracted from, the body by the exterior. In Section 3.1 the time derivative of

\footnotetext{
${ }^{1}$ For a history of the concept of force, see [Jammer 1999]. For earlier criticisms see [Mach 1883].

${ }^{2}$ See [Mach 1883, p. 83].

${ }^{3}$ See [Moreau 1970; 1974; Halphen and Son 1975; Germain et al. 1983].

${ }^{4}$ While the introduction of thermal variables reduces mechanics to a special case of thermodynamics, the approach based on dissipation potentials may lead to the opposite conclusion. For example, in a recently published theory of rigid heat conductors [Del Piero 2020], I consider temperature as a state variable in a purely mechanical context.
} 
the external energy, the external power, is assumed to be the sum of elementary powers, consisting of products of partial derivatives of the energy density, the external actions, classically subdivided into "distance" and "contact" actions, by the corresponding deformation rates. Section 3.2 contains Noll's deduction of Euler's balance equations of linear and angular momentum from the indifference of the external power to the representation of the body in the Euclidean space [Noll 1963]. In Section 3.3, the introduction of the cut principle of Euler and Cauchy leads to the definition of the stress tensor, along the line traced by Cauchy and completed by Noll. Though these are well known subjects, an innovative aspect is that, as already pointed out in [Del Piero 2019], the power associated with the stress is no longer viewed as an internal power, but as an alternative expression of the external power of the contact actions. ${ }^{5}$

Section 4 deals with two postulates, the conservation principle and the dissipation principle, proposed in [Del Piero 2019] as basic axioms of mechanics in place of Euler's balance laws. ${ }^{6}$ The conservation principle states that the external energy supplied to a body is entirely transformed into internal energy, and the dissipation principle says that a part of this energy is dissipated, that is, cannot be transformed back to external energy. In Section 4.1 the internal energy is assumed to be a function of two tensorial measures of macroscopic and microscopic deformation. From the theory of structured deformations come the results that the internal energy is the sum of two parts, one due to the microscopic deformation and one to the deformation generated by microdefects of various physical nature, collectively labeled as disarrangements, and that the latter is measured by the difference between macroscopic and microscopic deformation. ${ }^{7}$ In Section 4.2 the dissipation principle is used to subdivide the internal energy into two parts, reversible and dissipative. Both are supposed to have a volume density, which is a differentiable function for the reversible part and a dissipation potential for the dissipative part.

The conservation principle imposes the equality between internal and external power. Replacing the deformation rates by virtual velocities, the equation of virtual power is obtained in Section 4.3. This equation is of fundamental importance in mechanics, and was proposed as a basic postulate in place of Euler's balance laws. ${ }^{8}$ In Section 4.4 this equation is subjected to the Coleman-Noll procedure. Exploiting the arbitrariness of the virtual velocities, this procedure provides field equations and boundary conditions involving the external actions and the partial

${ }^{5}$ See the comment at the end of Section 3.3 below.

${ }^{6}$ Euler's laws lost the status of postulates after their deduction from the indifference of the external power.

${ }^{7}$ References and more information on structured deformations are given in the same Section 4.1.

${ }^{8}$ See, e.g., [Germain 1973a; 1973b]. In the present context this equation, being a consequence of the conservation principle, cannot be a postulate. 
derivatives of the internal energy densities. Finally, in Section 4.5 two types of dissipation potential, given by functions positively homogeneous of order one and of order two, are introduced. They correspond to the dissipative behavior observed in plastic and in viscous materials, respectively.

In Section 5, the field equations and boundary conditions obtained from the Coleman-Noll procedure are used to formulate the initial/boundary-value problem for two-scale continua. This problem takes different forms, depending on the form assumed for the dissipation potential. In the nondissipative case, the problem reduces to a family of independent equilibrium problems, one for each instant of time. In Section 5.1, the well-posedness of such problems is investigated for a simple one-dimensional model.

Section 5.2 deals with dissipation potentials of the viscous type. Such potentials are differentiable and rate-dependent, because the corresponding internal actions involve the time derivatives of the deformation. The initial/boundary-value problem reduces to an incremental problem, that is, to a differential problem in which at every instant the current deformation is known from the solution of the same problem at the preceding instants, and the unknown is the deformation rate. An elementary special case of this problem is the Kelvin-Voigt problem of linear viscoelasticity.

Being represented by homogeneous functions of order one, the dissipation potentials of the plastic type are rate-independent and nondifferentiable at the origin. For such potentials, as shown in Section 5.3, the formulation of the initial/boundaryvalue problem involves a nonsmooth flow rule and an activation condition for the plastic strain rate, which in plasticity is the requirement that the stress tensor be located on the boundary of the yield surface.

The final Section 5.4 is devoted to a comparison to Gurtin and Anand's theory of gradient plasticity. ${ }^{9}$ The comparison reveals both analogies and basic differences. A major difference is that, while Gurtin and Anand's approach involves additional stress measures, ${ }^{10}$ here the only stress measure is the Piola stress of classical continuum mechanics. The role of the additional stress measures is played by internal actions which, as said before, are partial derivatives of the internal energy densities which, in turn, are functions of the deformation. This avoids the vicious circle of first inventing new stress measures, and then inventing constitutive equations to relate them to the deformation.

${ }^{9}$ See [Gurtin and Anand 2005]. In earlier papers I frequently made comparisons to this theory, which, as far as I know, is the most advanced formulation of gradient plasticity. The theory of gradient plasticity was formulated by Aifantis [1984], and developed by Fleck and Hutchinson [1993] and others [Gudmundson 2004; Fleck and Willis 2009a; 2009b].

${ }^{10}$ The plastic stress and the hyperstress. Their necessity being considered as self-evident, they are introduced without any motivation or explanation. 
Classically, the constitutive equations convey the experimental information on the stress-strain response of specific materials. Here, this information is used to determine the shape of the internal energy. Only a single constitutive equation, relating the Piola stress to a specific combination of partial derivatives of the internal energy density, is required. Once the shape of the internal energy has been fixed, no further constitutive assumption is necessary.

\section{Two-scale representations of a physical body}

The physical bodies are objects located in the physical space. ${ }^{11}$ In classical mechanics the physical space is represented as a three-dimensional Euclidean point space $\mathscr{E}$, a physical body is represented as a region of $\mathscr{E}$ with the properties of a mathematical continuum, and its elementary constituents, the material points, are represented as points of that region. The representation preserves the distances between material points measured by an operator called the observer. A multiscale representation involves measurements made at scales of different orders of magnitude, macroscopic, mesoscopic, microscopic, submicroscopic, etc. Here we consider only two scales, macroscopic and microscopic. ${ }^{12}$

2.1. Placements and deformations. A second operator, the placer, "places" on $\mathscr{E}$ the material points, preserving the distances measured by the observer. ${ }^{13}$ Since the observer cannot measure an infinite number of distances, the placer can place only a finite set of material points. An image of the body as a continuum is then constructed by "filling" the interspaces between the placed points. ${ }^{14}$ A macroscopic placement of the body in $\mathscr{E}$ involves the following sequence of operations. ${ }^{15}$

(1) The observer measures the mutual distances in a selected set $\Xi^{N}$ made of $N$ material points.

(2) The placer places $\Xi^{N}$ into a set $\mathfrak{X}^{N}$ of $N$ points of $\mathscr{E}$, preserving the distances and the orientation. ${ }^{16}$

${ }^{11}$ Physical body and physical space are considered here as primitive, undefined concepts.

${ }^{12}$ In this case it is usual to speak of two-scale continua. This is done with some abuse of terminology, since being two-scale is a property of the representation of the body on $\mathscr{E}$ and not of the body itself, which by the way could also not be a continuum.

${ }^{13}$ An exact correspondence between material points and points of $\mathscr{E}$ is possible only if the physical space is metrizable with a Euclidean metric. See [Del Piero 2019, §2].

${ }^{14}$ This can be done both if the physical body is itself a continuum, and if it is made of a huge but finite number of material points, like in bodies with an atomic structure. Therefore, the "filling" operation does not imply any assumption about the structure of the physical body.

${ }^{15}$ All operations are discussed in detail in the paper [Del Piero 2019]. The description given here aims to render the present paper as self-contained as possible.

${ }^{16}$ The distance in $\mathfrak{X}^{N}$ is the Euclidean distance of $\mathscr{E}$. 
With the first operation, the observer induces on $\Xi^{N}$ a macroscopic distance function $d^{N}$. For the given $\Xi^{N}$, there is an infinity of sets $\mathfrak{X}^{N}$ in $\mathscr{E}$ which preserve the distances and the orientation. Each of them is a macroscopic placement of the metric space $\left(\Xi^{N}, d^{N}\right)$ on $\mathscr{E}$.

The distances $d^{N}$ may differ from one measurement to another. In this case, we say that the body is deformable. To represent a deformable body, it is convenient to fix once and for all a macroscopic reference placement $\mathfrak{X}_{R}^{N}$ of $\Xi^{N},{ }^{17}$ and to consider every other placement $\mathfrak{X}^{N}$ as the composition of the macroscopic reference map $\Xi^{N} \mapsto \mathfrak{X}_{R}^{N}$ with a discrete map $f_{R}^{N}: \mathfrak{X}_{R}^{N} \rightarrow \mathfrak{X}^{N}$. Then the passage to the continuum is made as follows.

(3) A continuous region $\Omega_{R}$ of $\mathscr{E}$ is constructed by "filling" the interspaces between the points of $\mathfrak{X}_{R}^{N}$.

(4) The discrete map $f_{R}^{N}$ from $\mathfrak{X}_{R}^{N}$ is extended to a map $f_{R}$ from $\Omega_{R}$.

The region $\Omega_{R}$ is not an exact representative of the body, because only the points of $\mathfrak{X}_{R}^{N}$ are images of material points. Nevertheless, $\Omega_{R}$ is taken as the macroscopic reference placement, that is, as the macroscopic representative, of the body in $\mathscr{E}$. The extended map $f_{R}$ is a macroscopic deformation from $\Omega_{R}$. This map is largely undetermined, the only condition dictated by physics being that $f_{R}$ be injective and orientation-preserving. ${ }^{18}$ For every $f_{R}$, the region $f_{R}\left(\Omega_{R}\right)$ is a macroscopic placement of the body. ${ }^{19}$

For the placement of a body at the microscopic scale, some additional operations are required.

(5) For each point $X^{\alpha}$ of $\Xi^{N}$, the observer selects a set $\Xi^{N_{\alpha}}$ of $N_{\alpha}$ material points close to $X^{\alpha}$, and measures their mutual distances. ${ }^{20}$

(6) The placer places each $\Xi^{N_{\alpha}}$ into a set $\mathfrak{X}^{N_{\alpha}}$ of $N_{\alpha}$ points of $\mathscr{E}$, preserving the distances and the orientation.

For each $\Xi^{N_{\alpha}}$, the distance measurements provide a microscopic distance function $d^{N_{\alpha}}$. Then $\mathfrak{X}^{N_{\alpha}}$ is a placement of the metric space $\left(\Xi^{N_{\alpha}}, d^{N \alpha}\right)$ on $\mathscr{E}$, and the set $\left\{\mathfrak{X}^{N},\left\{\mathfrak{X}^{N_{\alpha}}\right\}\right\}$ made of a placement of $\Xi^{N}$ and of the $N$ placements of the $\Xi^{N_{\alpha}}$ is a two-scale placement of $\left\{\Xi^{N},\left\{\Xi^{N_{\alpha}}\right\}\right.$. Every $\mathfrak{X}^{N_{\alpha}}$ can be viewed as the composition of a microscopic reference map from $\Xi^{N_{\alpha}}$ to a microscopic reference placement $\mathfrak{X}_{R}^{N_{\alpha}}$, followed by a map $f_{R}^{N_{\alpha}}$ from $\mathfrak{X}_{R}^{N_{\alpha}}$ to $\mathfrak{X}^{N_{\alpha}}$.

At the microscopic scale, the passage to the continuum is made as follows.

${ }^{17}$ The distances in $\mathfrak{X}_{R}^{N}$ may, or may not, correspond to measured distances in $\Xi^{N}$.

${ }^{18}$ The first condition excludes interpenetration of matter, and the second excludes placements which are the mirror images of each other.

${ }^{19}$ Different extensions $f_{R}$ of the same map $f_{R}^{N}$ are considered as different macroscopic deformations.

${ }^{20}$ It is assumed that for each $\alpha$ the set $\Xi^{N_{\alpha}}$ includes the point $X^{\alpha}$. 
(7) For each $X^{\alpha}$, the placer constructs a neighborhood $\mathcal{N}_{R}^{\alpha}$ of $\Omega_{R}$ by "filling" the interspaces between the points of the corresponding set $\mathfrak{X}_{R}^{N_{\alpha}}$.

(8) They then extend each discrete map $f_{R}^{N_{\alpha}}$ from $\mathfrak{X}_{R}^{N_{\alpha}}$ to a map $f_{R}^{\alpha}$ from $\mathcal{N}_{R}^{\alpha} \cdot{ }^{21}$

Taking the regions $\mathcal{N}_{R}^{\alpha}$ to be sufficiently small, each $f_{R}^{\alpha}$ is conveniently described by its gradient $\nabla f_{R}^{\alpha}$ at $x_{R}^{\alpha}$. Since all $f_{R}^{\alpha}$ are injective and orientation-preserving, the tensors

$$
F_{R}^{\alpha}=\nabla f_{R}^{\alpha}\left(x_{R}^{\alpha}\right), \quad \alpha \in\{1,2, \ldots, N\},
$$

belong to the space $\mathrm{Lin}^{+}$of all second-order tensors with positive determinant. The last step is the extension of the discrete set $\left\{F_{R}^{\alpha}\right\}$ to $\Omega_{R}$.

(9) The $N$ tensors $F_{R}^{\alpha}$ are extended to a continuous field $F_{R}$ over $\Omega_{R}$.

The map $F_{R}: \Omega_{R} \rightarrow \mathrm{Lin}^{+}$is a microscopic deformation from $\Omega_{R} \cdot{ }^{22}$ The pair $\left(f_{R}\left(\Omega_{R}\right), F_{R}\left(\Omega_{R}\right)\right)$ in the product space $\mathscr{E} \times \operatorname{Lin}^{+}$is a two-scale placement of the body, the pair $\left(f_{R}, F_{R}\right)$ is a two-scale deformation from $\Omega_{R}$, and the pair $\left(\nabla f_{R}\left(x_{R}\right), F_{R}\left(x_{R}\right)\right)$ is the local description of $\left(f_{R}, F_{R}\right)$ at $x_{R}$.

In a two-scale deformation from $\Omega_{R}$, a point $x_{R}$ of $\mathcal{N}_{R}^{\alpha}$ is mapped into the point $f_{R}\left(x_{R}\right)$ by the macroscopic deformation and into the point $f_{R}^{\alpha}\left(x_{R}\right)$ by the microscopic deformation. Then the vector $e_{R}=x_{R}-x_{R}^{\alpha}$ is mapped into $\nabla f_{R}\left(x_{R}^{\alpha}\right)\left[e_{R}\right]$ and into $F_{R}\left(x_{R}^{\alpha}\right)\left[e_{R}\right]$, respectively. The difference $\left(\nabla f_{R}\left(x_{R}^{\alpha}\right)-F_{R}\left(x_{R}^{\alpha}\right)\right)$ is a measure of the mismatch between the macroscopic and microscopic images of $\mathcal{N}_{R}^{\alpha}$. It will be called the distortion of the two-scale deformation at $x_{R}^{\alpha}$.

Two-scale deformations from any two-scale placement $\left(f_{R}\left(\Omega_{R}\right), F_{R}\left(\Omega_{R}\right)\right)$ can be defined using the fact that, due to the injectivity of $f_{R}$ and $F_{R}$, the inverse maps $f_{R}^{-1}$ and $F_{R}^{-1}$ exist. Then the inverse of $\left(f_{R}, F_{R}\right)$ is defined as the map $\left(f_{R}, F_{R}\right)^{-1}=\left(f_{R}^{-1}, F_{R}^{-1}\right)$ which maps the placement $\left(f_{R}\left(\Omega_{R}\right), F_{R}\left(\Omega_{R}\right)\right)$ back to $\Omega_{R}$. The composition

$$
(f, F)=\left(f_{R \varnothing}, F_{R \varnothing}\right) \circ\left(f_{R}, F_{R}\right)^{-1}
$$

is a deformation from $\left(f_{R}\left(\Omega_{R}\right), F_{R}\left(\Omega_{R}\right)\right)$ to $\left(f_{R \varnothing}\left(\Omega_{R}\right), F_{R \varnothing}\left(\Omega_{R}\right)\right)$. This deformation is injective, and its inverse is $\left(f_{R}, F_{R}\right) \circ\left(f_{R \varnothing}, F_{R \varnothing}\right)^{-1}$. Moreover, $(f, F)$ is orientation-preserving, because $f_{R}, F_{R}, f_{R \varnothing}$, and $F_{R \varnothing}$ are all orientation-preserving. Therefore, the set of all injective and orientation-preserving two-scale deformations $(f, F)$ has the algebraic structure of a group with respect to the composition (2-2).

${ }^{21}$ Like in the macroscopic case, the only condition required for the extended maps $f_{R}^{\alpha}$ is to be injective and orientation-preserving.

${ }^{22}$ The tensor field $F_{R}$ is not in general the gradient of a vector field. 
Additional restrictions can be imposed on "admissible" deformations with "nice" regularity properties, such as continuity or differentiability. ${ }^{23}$ In general, the restricted set of admissible deformations is required to preserve the group structure. For example, if the admissible deformations are continuous, their inverses must be continuous as well. That is, the admissible deformations must be homeomorphisms. If the admissible deformations are differentiable, the admissible deformations must be diffeomorphisms, that is, differentiable deformations with differentiable inverses.

2.2. Reference systems and reference frames. For each set of distance functions on $\left(\Xi^{N},\left\{\Xi^{N_{\alpha}}\right\}\right)$, there is an infinity of two-scale placements $\left(\mathfrak{X}^{N}, \mathfrak{X}^{N_{\alpha}}\right)$ in $\mathscr{E}$ which preserve distances and orientation. They form an equivalence class of two-scale placements called a two-scale configuration. A systematic way for selecting a representative element inside each equivalence class is to choose a reference system in the physical space, and a reference frame in $\mathscr{E}$.

A reference system is a discrete set $\left(X^{0}, X^{i}\right)$ of material points whose mutual distances are supposed to be the same in all distance measurements, ${ }^{24}$ and a reference frame is a distance- and orientation-preserving image $\left(x^{0}, x^{i}\right)$ of the reference system on $\mathscr{E} .{ }^{25}$ If the physical space can be represented as a three-dimensional Euclidean space, the number of the $X^{i}$ is three. Then in every distance measurement the position $x^{\alpha}$ in $\mathscr{E}$ of a material point $X^{\alpha}$ of $\Xi^{N}$ is determined uniquely by the linear combination

$$
x^{\alpha}-x^{0}=\sum_{i=1}^{3} \xi^{i} e^{i},
$$

with coefficients $\xi^{i}$ depending on the distances $\left|x^{\alpha}-x^{i}\right|$ and $\left|x^{\alpha}-x^{0}\right|,{ }^{26}$ and with the unit vectors

$$
e^{i}=\frac{x^{i}-x^{0}}{\left|x^{i}-x^{0}\right|}
$$

representing the directions in $\mathscr{E}$ associated with the reference frame. ${ }^{27}$

${ }^{23}$ In fact, regularity is assumed more for computational convenience than for physical reasons. For example, it is physically meaningless to require continuity for the deformations of a body with a discrete atomic structure.

24 "Like the walls of a laboratory, the fixed stars, or the wooden horses on a merry-go-round" [Truesdell and Noll 1965, §17].

${ }^{25}$ Less precise are the definitions given in the literature. For a comparison with the definitions of Truesdell and Noll [1965], see [Del Piero 2019, footnote 16].

${ }^{26}$ For their explicit form see [Del Piero 2019, §3.3]. An advantage of using a reference system is that, instead of measuring the mutual distances between all points $X^{\alpha}$ of $\Xi^{N}$, it is sufficient to measure the distances of each $X^{\alpha}$ from the points of the reference system.

${ }^{27}$ It may happen that, like in the case of the "fixed stars", the points $X^{i}$ are inaccessible to the observer. In this case, the observer is supposed to be able to recognize when three material points 
2.3. Two-scale evolutions. A two-scale evolution is a sequence of two-scale placements, ordered according to the flow of the physical time. In $\mathscr{E}$, a two-scale evolution is represented by a family $t \mapsto\left(f_{t}, F_{t}\right)$ of two-scale deformations from a fixed macroscopic reference placement $\Omega_{R}$. The parameter $t$, called the "time", need not coincide with the physical time, but must preserve the ordering established by the physical time.

An evolution is constructed starting from a set $\left(f_{t_{k}}, F_{t_{k}}\right)$ of two-scale deformations from the reference placement, relative to a finite number of instants $t_{k}$. With a "filling" procedure in time, this discrete set of deformations is extended to a finite time interval. The family $t \mapsto\left(f_{t}, F_{t}\right)$ is the representation on $\mathscr{E}$ of a two-scale evolution of the body. ${ }^{28}$

While the reference system is fixed once and for all, the reference frames may vary with $t$. Then let $t \mapsto\left(f_{t}, F_{t}\right)$ and $t \mapsto\left(f_{t}^{*}, F_{t}^{*}\right)$ be two representations of the same evolution relative to two families, $t \mapsto\left\{x_{t}^{0}, x_{t}^{i}\right\}$ and $t \mapsto\left\{x_{t}^{* 0}, x_{t}^{* i}\right\}$, of reference frames. For the macroscopic deformations $f_{t}, f_{t}^{*}$, let

$$
x_{t}^{*}=f_{t}^{*}\left(x_{R}\right), \quad x_{t}=f_{t}\left(x_{R}\right)
$$

be the trajectories of a point $x_{R}$ of $\Omega_{R}$ in the two representations. The distances $\left|x_{t}^{*}-x_{t}^{* 0}\right|$ and $\left|x_{t}-x_{t}^{0}\right|$ are equal, because both coincide with the distance of the material points $X, X^{0}$ measured at the time $t$. Then there is a family $t \mapsto Q_{t}$ of orthogonal tensors such that $\left(x_{t}^{*}-x_{t}^{* 0}\right)=Q_{t}\left[x_{t}-x_{t}^{0}\right]$, that is,

$$
f_{t}^{*}\left(x_{R}\right)=x_{t}^{* 0}+Q_{t}\left[f_{t}\left(x_{R}\right)-x_{t}^{0}\right] .
$$

This is the transformation rule for the macroscopic deformations under a change of reference frame. Moreover, differentiation with respect to $x_{R}$ provides the transformation rule

$$
\nabla f_{t}^{*}\left(x_{R}\right)=Q_{t} \nabla f_{t}\left(x_{R}\right)
$$

for the deformation gradients, and differentiation with respect to $t$ provides the transformation rule

$$
\dot{f}_{t}^{*}\left(x_{R}\right)=\dot{x}_{t}^{* 0}+Q_{t}\left[\dot{f}_{t}\left(x_{R}\right)-\dot{x}_{t}^{0}\right]+\dot{Q}_{t}\left[f_{t}\left(x_{R}\right)-x_{t}^{0}\right]
$$

for the time derivatives. ${ }^{29}$

are aligned. Then for each $X^{i}$ they select an accessible point $X^{i *}$ aligned with $X^{0}$ and $X^{i}$ and such that the distance between $X^{i *}$ and $X^{0}$ is the same in all distance measurements, and take $\left(X^{0}, X^{i *}\right)$ as the reference system.

${ }^{28}$ Just like the regularity in space, the assumed regularity in time may have no relation with physical reality.

${ }^{29}$ In the previous versions of the theory the time derivative was denoted by $\delta$, and the superimposed dot was reserved for the derivative with respect to the physical time. Here, to simplify the notation, we use the superimposed dot in both cases. 
For the microscopic deformations $F_{t}, F_{t}^{*}$, at every $t$ the lengths of the vectors $F_{t}\left(x_{R}\right)\left[e_{R}\right]$ and $F_{t}^{*}\left(x_{R}\right)\left[e_{R}\right]$ are the same for all $x_{R}$ and for all unit vectors $e_{R}$. Then at each $x_{R} \in \Omega_{R}$ there is an orthogonal tensor $Q_{x_{R} t}$ such that $F_{t}^{*}\left(x_{R}\right)=Q_{x_{R}} F_{t}\left(x_{R}\right)$. By the independence of the distortions on the reference frame, all $Q_{x_{R} t}$ must be equal to the tensor $Q_{t}$ in (2-7). ${ }^{30}$ Then we have

$$
F_{t}^{*}\left(x_{R}\right)=Q_{t} F_{t}\left(x_{R}\right)
$$

and, by time differentiation,

$$
\dot{F}_{t}^{*}\left(x_{R}\right)=Q_{t} \dot{F}_{t}\left(x_{R}\right)+\dot{Q}_{t} F_{t}\left(x_{R}\right) .
$$

We remark that $\dot{f}_{t}$ and $\dot{F}_{t}$ are not physical velocities, because the presence of $Q_{t}$, $x_{t}^{0}$, and $x_{t}^{* 0}$ and of their time derivatives makes them dependent on the variable reference frame.

\section{The external energy}

Classically, the deformations of a body are attributed to the action of physical agents, like contact with other bodies, gravitation, heat, and electric and magnetic fields. These agents are supposed to act through vectorial quantities called forces or actions, considered as physical entities. Today, a prevailing view is that a body deforms because it receives some energy from, or delivers some energy to, the exterior. In this view, energy is the primitive concept, and forces are derived quantities.

3.1. External energy and external power. In continuum mechanics it is generally assumed that there are two modes, at distance and by contact, for interchanging energy between a body and the exterior. In the present theory of two-scale continua, we assume that the external energy depends only on the current value $(f, F)$ of the two-scale deformation

$$
\mathscr{E}_{\mathrm{ext}}\left(\Omega_{R}, f, F\right)=\int_{\Omega_{R}} \beta\left(f\left(x_{R}\right), F\left(x_{R}\right)\right) \mathrm{dv}+\int_{\partial \Omega_{R}} \sigma\left(f\left(x_{R}\right), F\left(x_{R}\right)\right) \mathrm{da},
$$

thereby excluding the dependence on deformation gradients and on the past deformation history. We also assume that the volume density $\beta$ and the surface density $\sigma$ are differentiable functions. ${ }^{31}$ In an evolution $t \mapsto\left(f_{t}, F_{t}\right)$, the external power

$$
\mathscr{P}_{\text {ext }}\left(\Omega_{R}, \dot{f}_{t}, \dot{F}_{t}\right)=\int_{\Omega_{R}}\left(b_{R t} \cdot \dot{f}_{t}+B_{R t} \cdot \dot{F}_{t}\right) \mathrm{dv}+\int_{\partial \Omega_{R}}\left(s_{R t} \cdot \dot{f}_{t}+S_{R t} \cdot \dot{F}_{t}\right) \mathrm{da}
$$

\footnotetext{
${ }^{30}$ See [Del Piero 2019, §4.4].

${ }^{31}$ In this way we exclude, for example, the energy due to frictional contact.
} 
is obtained by time differentiation. The external actions, that is, the distance actions $b_{R t}, B_{R t}$ and the contact actions $s_{R t}, S_{R t}$, are the partial derivatives of $\beta$ and $\sigma$ at $\left(f_{t}, F_{t}\right) .{ }^{32}$

3.2. "Indifference principle" and balance laws. As already pointed out, $\dot{f}_{t}$ and $\dot{F}_{t}$ are not physical velocities, because they depend on the representation of the evolution on $\mathscr{E}$. It is just common sense to require that the power be independent of this representation. ${ }^{33}$ Therefore, if $t \mapsto\left(f_{t}, F_{t}\right)$ and $t \mapsto\left(f_{t}^{*}, F_{t}^{*}\right)$ are representations of the same evolution with respect to two different families of reference frames, not only are the derivatives $\dot{f}_{t}, \dot{F}_{t}$ and $\dot{f}_{t}^{*}, \dot{F}_{t}^{*}$ different, but also the external actions must transform in such a way that the resulting powers be the same. In particular, for the macroscopic power we have the indifference condition

$$
\int_{\Omega_{R}} b_{R t}^{*} \cdot \dot{f}_{t}^{*} \mathrm{dv}+\int_{\partial \Omega_{R}} s_{R t}^{*} \cdot \dot{f}_{t}^{*} \mathrm{da}=\int_{\Omega_{R}} b_{R t} \cdot \dot{f}_{t} \mathrm{dv}+\int_{\partial \Omega_{R}} s_{R t} \cdot \dot{f}_{t} \mathrm{da},
$$

and from the transformation law (2-8) for $\dot{f}_{t}$, we get

$$
\begin{aligned}
\int_{\Omega_{R}}\left(b_{R t}^{*} \cdot\right. & \left.\left(\dot{x}_{t}^{* 0}+Q_{t}\left[\dot{f}_{t}-\dot{x}_{t}^{0}\right]+\dot{Q}_{t}\left[f_{t}-x_{t}^{0}\right]\right)-b_{R t} \cdot \dot{f}_{t}\right) \mathrm{dv} \\
& +\int_{\partial \Omega_{R}}\left(s_{R t}^{*} \cdot\left(\dot{x}_{t}^{* 0}+Q_{t}\left[\dot{f}_{t}-\dot{x}_{t}^{0}\right]+\dot{Q}_{t}\left[f_{t}-x_{t}^{0}\right]\right)-s_{R t} \cdot \dot{f}_{t}\right) \mathrm{da}=0,
\end{aligned}
$$

for all $x_{t}^{* 0}, \dot{x}_{t}^{* 0}, Q_{t}, \dot{Q}_{t}$ and for all $\dot{f}_{t}{ }^{34}$ In particular, for $\dot{x}_{t}^{* 0}=\dot{Q}_{t}=0$, from the arbitrariness of $\dot{f}_{t}$ we deduce the transformation laws of the external actions

$$
b_{R t}^{*}=Q_{t} b_{R t}, \quad s_{R t}^{*}=Q_{t} s_{R t} .
$$

Substituting into (3-4), all terms involving $\dot{f}_{t}$ cancel. Then after setting $\dot{Q}_{t}=Q_{t} Y_{t}$ and $a_{t}=\left(Q_{t}^{T} \dot{x}_{t}^{* 0}-\dot{x}_{t}^{0}\right)$, it remains that

$$
\int_{\Omega_{R}} b_{R t} \cdot\left(a_{t}+Y_{t}\left[f_{t}-x_{t}^{0}\right]\right) \mathrm{dv}+\int_{\partial \Omega_{R}} s_{R t} \cdot\left(a_{t}+Y_{t}\left[f_{t}-x_{t}^{0}\right]\right) \mathrm{da}=0 .
$$

Moreover, by differentiation of $Q_{t}^{T} Q_{t}=I$ we have the relation

$$
\dot{Q}_{t}^{T} Q_{t}+Q_{t}^{T} \dot{Q}_{t}=0
$$

${ }^{32}$ Here $b_{R t} \cdot \dot{f}_{t}$ is a simplified notation for $\left(\beta_{f}\left(f_{t}\left(x_{R}\right), F_{t}\left(x_{R}\right)\right) \cdot \dot{f}_{t}\left(x_{R}\right)\right)$, and so on.

${ }^{33}$ In the past, this requirement was given the status of a basic principle. For a history of this "principle of material frame indifference" see [Truesdell and Noll 1965, §19A]. See also the remarks in [Murdoch 2012, §12.4]. Only later did Noll recognize the more modest nature of this requirement: "the principle of material frame-indifference is not a law of physics... it is merely a prescription for avoiding nonsense" [Noll 2006].

${ }^{34}$ Indeed, (3-4) holds for all evolutions from the same deformation $f_{t}$. 
which shows that $Y_{t}=Q_{t}^{T} \dot{Q}_{t}$ is a skew-symmetric tensor. Since $a_{t}$ and $Y_{t}$ are mutually independent, (3-6) splits into the separate conditions ${ }^{35}$

$$
\begin{aligned}
a \cdot\left(\int_{\Omega_{R}} b_{R} \mathrm{dv}+\int_{\partial \Omega_{R}} s_{R} \mathrm{da}\right) & =0, \\
Y \cdot\left(\int_{\Omega_{R}} b_{R} \otimes\left(f-x^{0}\right) \mathrm{dv}+\int_{\partial \Omega_{R}} s_{R} \otimes\left(f-x^{0}\right) \mathrm{da}\right) & =0 .
\end{aligned}
$$

Denoting by $y$ the vector associated with $Y$ by the identity

$$
Y \cdot(a \otimes b)=y \cdot b \times a \quad \text { for all } a, b \in \mathscr{V},
$$

and using the arbitrariness of $a$ and $y,(3-8)$ reduces to

$$
\begin{array}{r}
\int_{\Omega_{R}} b_{R} \mathrm{dv}+\int_{\partial \Omega_{R}} s_{R} \mathrm{da}=0, \\
\int_{\Omega_{R}}\left(f-x^{0}\right) \times b_{R} \mathrm{dv}+\int_{\partial \Omega_{R}}\left(f-x^{0}\right) \times s_{R} \mathrm{da}=0 .
\end{array}
$$

These are the balance laws of linear and angular momentum. For a long time, they have been considered the basic axioms of mechanics. In reality, as shown above, they are consequences of the indifference condition (3-3). ${ }^{36}$

For the microscopic external power the indifference condition is

$$
\int_{\Omega_{R}} B_{R t}^{*} \cdot \dot{F}_{t}^{*} \mathrm{dv}+\int_{\partial \Omega_{R}} S_{R t}^{*} \cdot \dot{F}_{t}^{*} \mathrm{da}=\int_{\Omega_{R}} B_{R t} \cdot \dot{F}_{t} \mathrm{dv}+\int_{\partial \Omega_{R}} S_{R t} \cdot \dot{F}_{t} \mathrm{da},
$$

where, by (2-10), $\dot{F}_{t}^{*}=Q_{t}\left(\dot{F}_{t}+Y_{t} F_{t}\right)$, with $Q_{t}$ and $Y_{t}$ the same orthogonal and skew-symmetric tensors as above by the independence of the distortions on the reference frame. In particular, for $Y=0$ we have

$$
\int_{\Omega_{R}}\left(Q_{t}^{T} B_{R t}^{*}-B_{R t}\right) \cdot \dot{F}_{t} \mathrm{dv}+\int_{\partial \Omega_{R}}\left(Q_{t}^{T} S_{R t}^{*}-S_{R t}\right) \cdot \dot{F}_{t} \mathrm{da}=0
$$

and since this must hold for all $\dot{F}_{t}$, the transformation laws

$$
B_{R t}^{*}=Q_{t} B_{R t}, \quad S_{R t}^{*}=Q_{t} S_{R t}
$$

${ }^{35}$ The powers on the left-hand side are those of the macroscopic rigid translations and of the macroscopic rigid rotations, respectively. For this reason, the indifference principle was also called the principle of independence of power on superimposed rigid motions [Green and Rivlin 1957]. Since both equations hold for every evolution and for all times, the subscript $t$ has been omitted.

${ }^{36}$ The balance laws are due to Euler, and their deduction from the indifference of the external power is due to Noll [1963]. It is surprising that such physically relevant conditions come from a requirement on the representation of the evolutions on $\mathscr{E}$. 
follow. Substituting into (3-12) we get the condition

$$
Y_{t} \cdot\left(\int_{\Omega_{R}} B_{R t} F_{t}^{T} \mathrm{dv}+\int_{\partial \Omega_{R}} S_{R t} F_{t}^{T} \mathrm{da}\right)=0,
$$

which is the microscopic counterpart of the balance law (3-10) $)_{2}$ of macroscopic angular momentum. Since there is no counterpart to (3-10), this condition does not admit a local form.

3.3. The cut principle and the stress tensor. It is currently assumed that contact actions $s_{\partial \Pi_{R}}$ of the same nature as the contact actions $s_{R}$ act at every internal surface of $\Omega_{R}$, and that an external power of the form (3-2) holds for every part $\Pi_{R}$ of $\Omega_{R}$. This is the cut principle of Euler and Cauchy. ${ }^{37}$

For a fixed $t$, consider a family $\varepsilon \mapsto \Pi_{\varepsilon}=\varepsilon \Pi_{R}$ of homothetic regions, and for each $\Pi_{\varepsilon}$ write the balance law (3-10) ${ }_{1}$. Assuming the uniform boundedness of the distance action $b_{R}$, when $\varepsilon \rightarrow 0$ the volume integral tends to zero faster than the area of $\partial \Pi_{\varepsilon}$. Then the area integral also tends to zero faster than $a\left(\partial \Pi_{\varepsilon}\right)$ :

$$
\lim _{\varepsilon \rightarrow 0^{+}} \frac{1}{\mathrm{a}\left(\partial \Pi_{\varepsilon}\right)} \int_{\partial \Pi_{\varepsilon}} s_{\partial \Pi_{\varepsilon}} \mathrm{da}=0 .
$$

Taking regions $\Pi_{R}$ of particular shapes, it has been proved that the contact action $s_{\partial \Pi_{R}}$ at a boundary point $x_{R}$ of $\Pi_{R}$ depends only on the exterior unit normal $n_{R}$ to $\partial \Pi_{R}$ at $x_{R}$, and that this dependence is linear. That is, there are a vector-valued map $\tilde{s}$ over $\Omega_{R} \times \mathscr{V}$ and a tensor field $T_{R}$ over $\Omega_{R}$ such that

$$
s_{\partial \Pi_{R}}\left(x_{R}\right)=\tilde{s}\left(x_{R}, n_{R}\right)=T_{R}\left(x_{R}\right) n_{R}
$$

for all regions $\Pi_{R}$ for which $x_{R}$ is a boundary point. The tensor $T_{R}\left(x_{R}\right)$ is the Piola stress at $x_{R} \cdot{ }^{38}$

From $(3-10)_{1}$ and from the divergence theorem we get

$$
\int_{\Omega_{R}}\left(\operatorname{div} T_{R}+b_{R}\right) \mathrm{dv}=0 .
$$

By the cut principle, this equation holds for every part $\Pi_{R}$ of $\Omega_{R}$. Then due to the arbitrariness of $\Pi_{R}$ the punctual equation

$$
\operatorname{div} T_{R}+b_{R}=0
$$

${ }^{37}$ See, e.g., [Truesdell 1991, p. 154]. This "principle" excludes the possibility that the contact actions at the interior surfaces of the body and those at the physical boundary be of a different nature. In particular, it excludes the presence of material surfaces, in the sense of Gurtin and Murdoch [1975], at the body's interior. This implies, for example, the absence of surface tension at the interior surfaces.

${ }^{38}$ The existence of the map $\hat{s}$, formerly a conjecture of Cauchy, was proved later by Noll [1959], and the existence of $T_{R}$ is proved in Cauchy's tetrahedron theorem. 
holds at almost every point of $\Omega_{R}$. This is the local form of the balance law of linear momentum. In a similar way, from the divergence theorem applied to the indifference condition (3-8) 2 we get the punctual condition

$$
Y \cdot T_{R} \nabla f^{T}=0,
$$

which shows that the local form of the balance law of linear momentum is the requirement that the Cauchy stress tensor $\left(\operatorname{det} \nabla f^{-1}\right) T_{R} \nabla f^{T}$ be symmetric.

From (3-16) and (3-18) and from the divergence theorem we have

$$
\int_{\Pi_{R}} T_{R} \cdot \nabla \dot{f} \mathrm{dv}=\int_{\Pi_{R}} b_{R} \cdot \dot{f} \mathrm{dv}+\int_{\partial \Pi_{R}} s_{\partial \Pi_{R}} \cdot \dot{f} \mathrm{da},
$$

and substitution into (3-2) provides the reduced form of the external power

$$
\mathscr{P}_{\text {ext }}\left(\Omega_{R}, \dot{f}, \dot{F}\right)=\int_{\Omega_{R}}\left(T_{R} \cdot \nabla \dot{f}+B_{R} \cdot \dot{F}\right) \mathrm{dv}+\int_{\partial \Omega_{R}} S_{\partial \Pi_{R}} \cdot \dot{F} \mathrm{da}
$$

The traditional interpretation of (3-20) is that $T_{R}$ is an internal action, $T_{R} \cdot \nabla \dot{f}$ is an internal power, and the equation is an equality between internal and external power. ${ }^{39}$ In the present context this interpretation is incorrect because (3-20), being a consequence of (3-16) and (3-18), is an identity between two alternative forms of the external power. Therefore, the stress $T_{R}$ is not an internal action, but a tensorial representative of the external contact action acting on $\partial \Pi_{R}$.

3.4. Inertia. When taking energy as a primitive concept, a natural starting point for describing the phenomenon of inertia is to define the kinetic energy. Let $\rho$ be the mass density of the body in the reference placement $\Omega_{R} \cdot{ }^{40}$ Suppose that the body interacts with a second body, and consider an evolution $t \mapsto f_{t}$ of the two bodies. The kinetic energy at a point $x_{R}$ of the first body due to the interaction with the second body is an external energy with volume density

$$
\beta^{\mathrm{kin}}\left(f_{t}\left(x_{R}\right)\right)=-\frac{1}{2} \rho\left(x_{R}\right)\left|\dot{f}_{t}\left(x_{R}\right)-\dot{f}_{t}\left(x_{\varnothing R}\right)\right|^{2},
$$

where $t$ is the physical time, $x_{\varnothing R}$ is the position of the center of mass of the second body in the reference placement, and $\left(\dot{f}_{t}\left(x_{R}\right)-\dot{f}_{t}\left(x_{\varnothing R}\right)\right)$ is the velocity of the point

${ }^{39}$ See [Germain 1973a; 1973b; Truesdell 1991, §1.15; Gurtin et al. 2010, §19.7.2].

${ }^{40}$ The concept of mass, generally considered as a fundamental ingredient for mechanics, is in fact relevant only when inertia is taken into account; see the comment of Noll [2004]. The same author wrote that "the basic concepts of mechanics in general should not include items such as momentum, kinetic energy, and angular momentum, because they are relevant only when inertia is important" [Noll 1995]. 
$x_{t}=f_{t}\left(x_{R}\right)$ relative to the point $x_{\varnothing t}=f_{t}\left(x_{\varnothing_{R}}\right){ }^{41}$ The external power

$$
\dot{\beta}^{\mathrm{kin}}\left(f_{t}\left(x_{R}\right)\right)=-\rho\left(x_{R}\right)\left(\ddot{x}_{t}-\ddot{x}_{\varnothing t}\right) \cdot\left(\dot{x}_{t}-\dot{x}_{\varnothing t}\right)
$$

is the inner product of the inertial action

$$
b_{R t}^{\mathrm{kin}}\left(x_{R}\right)=-\rho\left(x_{R}\right)\left(\ddot{x}_{t}-\ddot{x}_{\varnothing t}\right)
$$

with the relative velocity $\left(\dot{x}_{t}-\dot{x}_{\varnothing t}\right) .^{42}$

Traditionally, the kinetic energy is written with $\dot{x}_{t}$ in place of $\left(\dot{x}_{t}-\dot{x}_{\varnothing t}\right)$, with the specification that the inertia law $b_{R t}^{\mathrm{kin}}=-\rho \ddot{x}_{t}$ holds only for a privileged class of observers, those moving with a uniform rectilinear motion with respect to the fixed stars. ${ }^{43}$ As pointed out in [Truesdell and Noll 1965, §18], this view is based on the Newtonian idea that the physical space is an absolute space, in which each body particle occupies a precise position, and that the "fixed stars" occupy a fixed position in that space. In particular, the point $x_{\varnothing R}$, which is identified with the center of mass of the universe, is assumed to have a fixed position in the absolute space, so that $\dot{x}_{\varnothing t}=\ddot{x}_{\varnothing t}=0$.

While the Euclidean point space is indeed an absolute space, in the physical space there are no absolute positions, but only positions relative to a given reference system. ${ }^{44}$ And since there are no absolute positions, whether or not an observer is "moving", whatever this means, is a false question. ${ }^{45}$

\section{The internal energy}

4.1. The two basic axioms. A physical body is supposed to have an internal energy. In a two-scale continuum, this energy depends on the local two-scale deformation $(\nabla f, F)$ and possibly on some additional state variables. ${ }^{46}$ In the simple

\footnotetext{
${ }^{41}$ It is perfectly conceivable to consider $\beta^{\text {kin }}$ as a function of the two-scale deformation $\left(f_{t}, F_{t}\right)$, including a microkinetic energy depending on $F_{t}$, like in [Capriz 1989, §7].

${ }^{42}$ The idea that the "inertia forces" are particular external actions is due to d'Alembert. According to Noll [1963], “...l'on regarde les forces d'inertie comme des forces véritables qui sont les interactions entre les corps dans notre système solaire et la totalité des objets dans le reste de l'univers".

${ }^{43}$ On the contrary, in the kinematics developed in [Del Piero 2019] the only operation made by the observer is the measurement of distances. Then it makes no sense to consider more than one observer or, even worse, to assume there are "privileged" observers.

${ }^{44}$ The conception of the physical space as an absolute space has unpleasant consequences, like the belief in the existence of the "luminiferous aether" and of mysterious "apparent forces". This conception was repeatedly criticized over the centuries; see, e.g., [Mach 1883, p. 543]. For further references see [Del Piero 2019, §8.5]. On the contrary, if the kinetic energy is defined as in (3-22) no apparent forces are required because relative instead of absolute velocities are considered.

${ }^{45}$ What are "moving" with respect to each other are the reference frames, and "apparent forces" appear if their relative motions, which are arbitrarily chosen by the placer, are neglected.

${ }^{46}$ For example, thermal, electromagnetic, or chemical variables.
} 
model developed here there are no state variables, and the energy is supposed to be the sum of a recoverable and of a dissipative part

$$
\mathscr{E}_{\text {int }}\left(\Omega_{R}, f, F\right)=\mathscr{E}_{\text {int }}^{\text {rec }}\left(\Omega_{R}, f, F\right)+\mathscr{E}_{\text {int }}^{\text {dis }}\left(\Omega_{R}, f, F\right),
$$

where the recoverable part is a volume integral with volume density depending on the current values of the local two-scale deformation and of its spatial gradients

$$
\mathscr{E}_{\text {int }}^{\mathrm{rec}}\left(\Omega_{R}, f, F\right)=\int_{\Omega_{R}} \Phi\left(\nabla f, F, \nabla^{2} f, \nabla F\right) \mathrm{dv},
$$

and in every deformation process $\tau \mapsto\left(f_{\tau}, F_{\tau}\right)$ from $\tau=0$ to $\tau=t$ the dissipative part has the form

$$
\underset{\mathscr{C}_{\text {int }}}{\mathscr{C i s}_{\mathrm{dis}}}\left(\Omega_{R}, f_{t}, F_{t}\right)=\mathscr{E}_{\mathrm{int}}^{\mathrm{dis}}\left(\Omega_{R}, f_{0}, F_{0}\right)+\int_{\Omega_{R}} \int_{t_{0}}^{t} \chi\left(\nabla \dot{f}_{\tau}, \dot{F}_{\tau}, \nabla^{2} \dot{f}_{\tau}, \nabla \dot{F}_{\tau}\right) d \tau \mathrm{dv}
$$

The relation between external and internal energy is subjected to two basic postulates. In classical mechanics, the role of postulates is played by the balance laws of Euler or, alternatively, by the equation of virtual power. ${ }^{47}$ In the more general context assumed in [Del Piero 2019], the same role is attributed to two "principles" more in line with general physics, the conservation principle and the dissipation principle. $^{48}$

The conservation principle says that to every amount of external energy supplied by, or extracted from, the body by the exterior, corresponds an equal increase, or decrease, of internal energy. This principle is expressed by the power equation

$$
\mathscr{P}_{\text {ext }}\left(\Omega_{R}, \dot{f}, \dot{F}\right)=\mathscr{P}_{\text {int }}\left(\Omega_{R}, \dot{F}, \dot{F}^{d}\right) .
$$

The dissipation principle states that in every deformation process the dissipative part of the internal energy is nonnegative. This corresponds to assuming that the dissipation potential $\chi$ is nonnegative,

$$
\chi\left(\nabla \dot{f}_{\tau}, \dot{F}_{\tau}, \nabla^{2} \dot{f}_{\tau}, \nabla \dot{F}_{\tau}\right) \geq 0
$$

for whatever values of the variables $\nabla \dot{f}_{\tau}, \dot{F}_{\tau}, \nabla^{2} \dot{f}_{\tau}, \nabla \dot{F}_{\tau}$.

4.2. Disarrangements. For the dependence of the energy on two-scale deformations, a particular form based on the additive decomposition of the macroscopic deformation gradient

$$
\nabla f=F+F^{d}
$$

${ }^{47}$ For axiomatics founded on the equation of virtual power see [Germain 1973a; 1973b].

${ }^{48}$ The two principles can be regarded as mechanical versions of the two laws of thermodynamics. In fact, there are different opinions on whether the conservation principle was first formulated in mechanics and then extended to other branches of physics or vice versa; see the discussion in [Mach 1896, pp. 295-297]. 
was assumed in [Del Piero 2018c; 2019]. Though a multiplicative decomposition looks more appropriate to a context of large deformations, a strong motivation in favor of the additive decomposition is provided by the theory of structured deformations. ${ }^{49}$ In [Del Piero and Owen 1993] it was proved that every structured deformation is the uniform limit of sequences $n \mapsto f_{n}$ of piecewise continuous one-scale deformations, with a number of discontinuities tending to infinity and amplitudes tending to zero as $n \rightarrow \infty$. Subsequently, it was proved in [Del Piero and Owen 1995] that the difference $\nabla f-F$ between the gradient of the limit $f$ of the functions $f_{n}$ and the limit $F$ of the absolutely continuous parts of the gradients $\nabla f_{n}$ is the volume density of the total deformation due to the singular parts of the $\nabla f_{n}$, that is, to the microscopic disarrangements associated with the limit deformation $(f, F) .{ }^{50}$

In the energetics of structured deformations developed by Choksi and Fonseca [1997], the internal energy of a structured deformation was defined as the relaxed limit, that is, as the infimum of the energies of all approximating sequences. The main result of [Choksi and Fonseca 1997] was that the relaxed energy has a volume density $\Phi$ depending on the local deformation $(\nabla f, F)$. The paper ended with the conjecture that this energy should be the sum of two parts, the relaxed limits of the bulk and the interfacial part of the energies of the approximating sequences, and that the volume densities of the two parts should depend on $F$ and $F^{d}$, respectively: ${ }^{51}$

$$
\Phi(\nabla f, F)=\varphi(F)+\psi\left(F^{d}\right) .
$$

Later, this conjecture was proved to be true. ${ }^{52}$

Here, for the volume density $\Phi$ of the recoverable part (4-2) we assume the decomposition $^{53}$

$$
\Phi\left(\nabla f, F, \nabla^{2} f, \nabla F\right)=\varphi(F, \nabla F)+\psi\left(F^{d}, \nabla F^{d}\right),
$$

${ }^{49}$ For a comparison between additive and multiplicative decompositions in finite plasticity see the paper [Del Piero 2018b]. For the purposes of the present paper, structured deformations can be identified with two-scale deformations.

${ }^{50}$ In crystal plasticity, a phenomenological characterization of $(\nabla f-F)$ based on slip surfaces and dislocation structures has been given by Reina and Conti [2014].

${ }^{51}$ From the mechanical viewpoint this is very reasonable because, due to the indeterminacy inherent to the "filling" procedure, the macroscopic deformation $\nabla f$ seems unfit to characterize a strain energy. For the external energy, on the contrary, it seems more appropriate to keep the dependence on the pair $(\nabla f, F)$, whose values are deduced directly from distance measurements.

${ }^{52}$ See [Owen 2000] and the present author's [Del Piero 2001] for the one-dimensional case, and [Šilhavý 2017] for the full three-dimensional case.

${ }^{53}$ In [Barroso et al. 2017], the dependence of $\varphi$ on the pair $(F, \nabla F)$ was proved for the energy of three-scale deformations, also called second-order structured deformations. 
and for the dissipation potential (4-3) we assume the form

$$
\chi\left(\nabla \dot{f}_{t}, \dot{F}_{t}, \nabla^{2} \dot{f}_{t}, \nabla \dot{F}_{t}\right)=\chi\left(\dot{F}_{t}^{d}, \nabla \dot{F}_{t}^{d}\right),
$$

that is, we assume that the dissipation is entirely due to the disarrangements. Then in every evolution $t \mapsto\left(f_{t}, F_{t}\right)$ the internal power

$$
\mathscr{P}_{\text {int }}\left(\Omega_{R}, \dot{F}_{t}, \dot{F}_{t}^{d}\right)=\int_{\Omega_{R}}\left(\dot{\varphi}\left(F_{t}, \nabla F_{t}\right)+\dot{\psi}\left(F_{t}^{d}, \nabla F_{t}^{d}\right)+\chi\left(\dot{F}_{t}^{d}, \nabla \dot{F}_{t}^{d}\right)\right) \mathrm{dv}
$$

is obtained by time differentiation of (4-1).

An energy density is recoverable if it is differentiable. ${ }^{54}$ Then we assume that both $\varphi$ and $\psi$ are differentiable. For the dissipation potential $\chi$, we assume that it is a convex function, positive except at $\left(\dot{F}_{t}^{d}, \nabla \dot{F}_{t}^{d}\right)=(0,0)$, where $\chi(0,0)=0 .{ }^{55}$

4.3. The equation of virtual power. By the assumed differentiability of $\varphi$ and $\psi$, in the expression (4-10) of the internal power we have

$$
\begin{aligned}
\dot{\varphi}\left(F_{t}, \nabla F_{t}\right) & =\varphi_{F}\left(F_{t}, \nabla F_{t}\right) \cdot \dot{F}_{t}+\varphi_{\nabla F}\left(F_{t}, \nabla F_{t}\right) \cdot \nabla \dot{F}_{t}, \\
\dot{\psi}\left(F_{t}^{d}, \nabla F_{t}^{d}\right) & =\psi_{F^{d}}\left(F_{t}^{d}, \nabla F_{t}^{d}\right) \cdot \dot{F}_{t}^{d}+\psi_{\nabla F^{d}}\left(F_{t}^{d}, \nabla F_{t}^{d}\right) \cdot \nabla \dot{F}_{t}^{d},
\end{aligned}
$$

with $\varphi_{F}, \varphi_{\nabla F}$ and $\psi_{F^{d}}, \psi_{\nabla F^{d}}$ the partial derivatives of $\varphi$ and $\psi$. Equating the internal power (4-10) to the reduced external power (3-21) and recalling that $\nabla \dot{f}=$ $\dot{F}+\dot{F}^{d}$, the power equation (4-4) takes the form ${ }^{56}$

$$
\begin{array}{r}
\int_{\Omega_{R}}\left(\left(T_{R t}+B_{R t}-\varphi_{F_{t}}\right) \cdot \dot{F}_{t}-\varphi_{\nabla F_{t}} \cdot \nabla \dot{F}_{t}+\left(T_{R t}-\psi_{F_{t}^{d}}\right)\right. \\
\left.\cdot \dot{F}_{t}^{d}-\psi_{\nabla F_{t}^{d}} \cdot \nabla \dot{F}_{t}^{d}-\chi\left(\dot{F}_{t}^{d}, \nabla \dot{F}_{t}^{d}\right)\right) \mathrm{dv} \\
+\int_{\partial \Omega_{R}}\left(S_{R t} \cdot \dot{F}_{t}\right) \mathrm{da}=0, \quad(4-12)
\end{array}
$$

and applying the divergence theorem to the terms involving $\varphi_{\nabla F_{t}}$ and $\psi_{\nabla F_{t}^{d}}$ we get

$$
\begin{aligned}
\int_{\Omega_{R}}\left(\left(T_{R t}+B_{R t}\right) \cdot \dot{F}_{t}+T_{R t} \cdot \dot{F}_{t}^{d}\right) \mathrm{dv} & +\int_{\partial \Omega_{R}} S_{R t} \cdot \dot{F}_{t} \mathrm{da} \\
=\int_{\Omega_{R}}\left(\left(\varphi_{F_{t}}-\operatorname{div} \varphi_{\nabla F_{t}}\right) \cdot \dot{F}_{t}\right. & \left.+\left(\psi_{F_{t}^{d}}-\operatorname{div} \psi_{\nabla F_{t}^{d}}\right) \cdot \dot{F}_{t}^{d}+\chi\left(\dot{F}_{t}^{d}, \nabla \dot{F}_{t}^{d}\right)\right) \mathrm{dv} \\
& +\int_{\partial \Omega_{R}}\left(\varphi_{\nabla F_{t}} n_{R} \cdot \dot{F}_{t}+\psi_{\nabla F_{t}^{d}} n_{R} \cdot \dot{F}_{t}^{d}\right) \mathrm{da} .
\end{aligned}
$$

${ }^{54}$ Indeed, if $\varphi$ is differentiable, from the relation $\varphi\left(F_{t}, \nabla F_{t}\right)=\varphi\left(F_{0}, \nabla F_{0}\right)+\varphi_{F}\left(F_{0}, \nabla F_{0}\right)$. $\left(F_{t}-F_{0}\right)+\varphi_{\nabla F}\left(F_{0}, \nabla F_{0}\right) \cdot\left(\nabla F_{t}-\nabla F_{0}\right)+o\left(\left|\left(F_{t}-F_{0}\right)\right|,\left|\nabla F_{t}-\nabla F_{0}\right|\right)$, it follows that $\varphi\left(F_{t}, \nabla F_{t}\right)=$ $\varphi\left(F_{0}, \nabla F_{0}\right)$ in every evolution with $\left(F_{t}, \nabla F_{t}\right)=\left(F_{0}, \nabla F_{0}\right)$.

${ }^{55}$ This is the simplest way for making the dissipation dependent on the past history of the evolution $t \mapsto\left(F_{t}^{d}, \nabla F_{t}^{d}\right)$.

${ }^{56}$ Here $\varphi_{F_{t}}$ is an abbreviation for $\varphi_{F}\left(F_{t}, \nabla F_{t}\right)$, and so on. 
For a given evolution $t \mapsto\left(f_{t}, F_{t}\right)$, consider the perturbed evolution starting at the instant $t_{0}$

$$
f_{\varepsilon t}=f_{t}+\varepsilon\left(t-t_{0}\right) v, \quad F_{\varepsilon t}=F_{t}+\varepsilon\left(t-t_{0}\right) V,
$$

with $v$ and $V$ arbitrary vector and second-order tensor fields. By time differentiation, we get the perturbed velocities

$$
\dot{F}_{\varepsilon t}=\dot{F}_{t}+\varepsilon V, \quad \dot{F}_{\varepsilon t}^{d}=\dot{F}_{t}^{d}+\varepsilon V^{d},
$$

where $V^{d}=\nabla v-V$. Writing (4-13) for the perturbed evolution and subtracting the same equation for the unperturbed evolution, the equation of virtual power

$$
\begin{aligned}
\int_{\Omega_{R}}\left(\left(T_{R t}+B_{R t}\right) \cdot V+T_{R t} \cdot V^{d}\right) \mathrm{dv} & +\int_{\partial \Omega_{R}} S_{R t} \cdot V \mathrm{da} \\
=\int_{\Omega_{R}}\left(\left(\varphi_{F_{t}}-\operatorname{div} \varphi \varphi_{\nabla}\right) \cdot V+\left(\psi_{F_{t}^{d}}\right.\right. & \left.\left.-\operatorname{div} \psi_{\nabla F_{t}^{d}}\right) \cdot V^{d}+\delta \chi\left(\dot{F}_{t}^{d}, \nabla \dot{F}_{t}^{d}, V^{d}, \nabla V^{d}\right)\right) \mathrm{dv} \\
& +\int_{\partial \Omega_{R}}\left(\varphi_{\nabla F_{t}} n_{R} \cdot V+\psi_{\nabla F_{t}^{d}} n_{R} \cdot V^{d}\right) \mathrm{da}
\end{aligned}
$$

is obtained, where

$$
\begin{aligned}
\delta \chi\left(\dot{F}_{t}^{d}, \nabla \dot{F}_{t}^{d}, V^{d},\right. & \left.\nabla V^{d}\right) \\
& =\lim _{\varepsilon \rightarrow 0} \frac{1}{\varepsilon}\left(\chi\left(\dot{F}_{t}^{d}+\varepsilon V^{d}, \nabla \dot{F}_{t}^{d}+\varepsilon \nabla V^{d}\right)-\chi\left(\dot{F}_{t}^{d}, \nabla \dot{F}_{t}^{d}\right)\right) .
\end{aligned}
$$

The equation of virtual power can be viewed as a purely mechanical version of the Clausius-Duhem inequality. ${ }^{57}$ In the more general context of thermodynamics, this inequality was exploited by Coleman and Noll to get restrictions on the form of the constitutive equations. ${ }^{58}$ On the contrary, in the present purely mechanical context the Coleman-Noll procedure will be used to deduce the field equations and boundary conditions for the initial/boundary-value problem.

4.4. The Coleman-Noll procedure. Consider first a purely macroscopic perturbation, in which $V^{d}=0$ and $V$ is an arbitrary second-order tensor field. Due to the arbitrariness of $V$, from (4-16) we get the local conditions

$$
\begin{aligned}
\varphi_{F_{t}}-\operatorname{div} \varphi_{\nabla F_{t}} & =T_{R t}+B_{R t} & & \text { in } \Omega_{R}, \\
S_{R t} & =\varphi_{\nabla F_{t}} n_{R} & & \text { on } \partial \Omega_{R} .
\end{aligned}
$$

${ }^{57}$ Called the free-energy imbalance in [Gurtin et al. 2010, §29]. Here the inequality reduces to equality because the whole dissipation is supposed to be due to the dissipation potential $\chi$.

${ }^{58}$ See [Coleman and Noll 1963]. 
Equation (4-16) then reduces to

$$
\begin{aligned}
\int_{\Omega_{R}}\left(\left(T_{R t}-\psi_{F_{t}^{d}}+\operatorname{div} \psi_{\nabla F_{t}^{d}}\right) \cdot V^{d}-\delta \chi\left(\dot{F}_{t}^{d}, \nabla \dot{F}_{t}^{d}, V^{d}, \nabla V^{d}\right)\right) \mathrm{dv} & \\
& -\int_{\partial \Omega_{R}} \psi_{\nabla F_{t}^{d}} n_{R} \cdot V^{d} \mathrm{da}=0 .
\end{aligned}
$$

In particular, taking $V^{d}$ with support on a ball of radius $\varepsilon$ centered first at an interior point and then at a boundary point of $\Omega_{R}$, we get the local conditions

$$
\begin{aligned}
\left(T_{R t}-\psi_{F_{t}^{d}}+\operatorname{div} \psi_{\nabla F_{t}^{d}}\right) \cdot V^{d}-\delta \chi\left(\dot{F}_{t}^{d}, \nabla \dot{F}_{t}^{d}, V^{d}, \nabla V^{d}\right)=0 & \text { in } \Omega_{R}, \\
\left(\psi_{\nabla F_{t}^{d}} n_{R}\right) \cdot V^{d}=0 & \text { on } \partial \Omega_{R} .
\end{aligned}
$$

These conditions cannot be further refined, as long as the term $\delta \chi$ remains unspecified. The forms taken by $\delta \chi$ for some special forms of the dissipation potential $\chi$ are specified in the next section.

4.5. Special forms of the dissipation potential. According to the definition given in Section 4.2, a dissipation potential is a convex function of the pair $\left(\dot{F}^{d}, \nabla \dot{F}^{d}\right)$, positive except at $(0,0)$, and with $\chi(0,0)=0$. In the literature we find dissipation potentials of two types, positively homogeneous of order one and of order two. ${ }^{59}$ In what follows we deal with such dissipation potentials, and we initially consider functions $\chi$ independent of $\nabla \dot{F}^{d}$.

A positively homogeneous $\chi$ of order two and differentiable at $\dot{F}^{d}=0$ has the form

$$
\chi\left(\dot{F}^{d}\right)=\frac{1}{2} \chi_{\dot{F}^{d} \dot{F}^{d}}(0)\left[\dot{F}^{d}\right] \cdot \dot{F}^{d},
$$

and after some routine computation, from (4-18) we get

$$
\delta \chi\left(\dot{F}^{d}, V^{d}\right)=\chi_{\dot{F}^{d} \dot{F}^{d}}(0)\left[\dot{F}^{d}\right] \cdot \dot{V}^{d} .
$$

A positively homogeneous $\chi$ of order one cannot be differentiable, but only directionally differentiable at $\dot{F}^{d}=0$. In this case it has the form

$$
\chi\left(\dot{F}^{d}\right)=\breve{\chi}_{\dot{F}^{d}}(0) \triangleright \dot{F}^{d},
$$

where $\breve{\chi}_{\dot{F}^{d}}(0) \triangleright V^{d}$ denotes the directional derivative of $\chi$ at zero in the direction $\dot{F}^{d}{ }^{60}$ From convexity, we have

$$
\chi\left(\frac{1}{2}\left(\dot{F}^{d}+\varepsilon V^{d}\right)\right) \leq \frac{1}{2}\left(\chi\left(\dot{F}^{d}\right)+\chi\left(\varepsilon V^{d}\right)\right),
$$

${ }^{59}$ See, e.g., [Ziegler 1963; Moreau 1970; Hackl and Fischer 2008].

${ }^{60}$ This notation was introduced in [Del Piero 2018c]. An example of a dissipation potential of this form is $\chi\left(\dot{F}^{d}\right)=k \mid \dot{F}^{d}$, with $k$ a positive constant. 
with equality for $V^{d}=\dot{F}^{d}$, and from homogeneity it follows that

$$
\chi\left(\dot{F}^{d}+\varepsilon V^{d}\right) \leq \chi\left(\dot{F}^{d}\right)+\varepsilon \chi\left(V^{d}\right),
$$

with equality for $V^{d}=\dot{F}^{d}$. Then, by (4-18), (4-23), and (4-25),

$$
\delta \chi\left(\dot{F}^{d}, V^{d}\right)=\lim _{\varepsilon \rightarrow 0} \frac{1}{\varepsilon}\left(\chi\left(\dot{F}^{d}+\varepsilon V^{d}\right)-\chi\left(\dot{F}^{d}\right)\right) \leq \chi\left(V^{d}\right)=\breve{\chi}_{\dot{F}^{d}}(0) \triangleright V^{d},
$$

with equality for $V^{d}=\dot{F}^{d}$.

Let us now consider dissipation potentials depending on both $\dot{F}^{d}$ and $\nabla \dot{F}^{d}$, of the special form

$$
\chi\left(\dot{F}^{d}, \nabla \dot{F}^{d}\right)=\chi^{\prime}\left(\dot{F}^{d}\right)+\chi^{\prime \prime}\left(\nabla \dot{F}^{d}\right),
$$

with both $\chi^{\prime}$ and $\chi^{\prime \prime}$ positive, convex, and differentiable everywhere except at $\dot{F}^{d}=0$ and $\nabla \dot{F}^{d}=0$, respectively. It can be easily verified that if both $\chi^{\prime}$ and $\chi^{\prime \prime}$ are positively homogeneous of order two, then

$$
\delta \chi\left(\dot{F}^{d}, \nabla \dot{F}_{t}^{d}, V^{d}, \nabla V^{d}\right)=\chi_{\dot{F}^{d} \dot{F}^{d}}^{\prime}(0)\left[\dot{F}^{d}\right] \cdot V^{d}+\chi_{\nabla \dot{F}^{d} \nabla \dot{F}^{d}}^{\prime \prime}(0)\left[\nabla \dot{F}^{d}\right] \cdot \nabla V^{d},
$$

and that if both are positively homogeneous of order one, then

$$
\delta \chi\left(\dot{F}^{d}, \nabla \dot{F}_{t}^{d}, V^{d}, \nabla V^{d}\right) \leq \breve{\chi}_{\dot{F}^{d}}^{\prime}(0) \triangleright V^{d}+\breve{\chi}_{\nabla \dot{F}^{d}}^{\prime \prime}(0) \triangleright \nabla V^{d},
$$

with equality for $V^{d}=\dot{F}^{d}$ and $\nabla V^{d}=\nabla \dot{F}^{d}$.

\section{Initial/boundary-value problems}

Equations (4-18) and (4-20) are the result of the Coleman-Noll procedure applied to the present model. On them and on the indifference conditions (3-10) and (3-14) is based the formulation of the initial/boundary-value problems for two-scale continua. To begin, we consider the case without dissipation.

5.1. The nondissipative problem. If there is no dissipation, then $\chi=\delta \chi=0$ and, since $V^{d}$ is allowed to be any second-order tensor, conditions (4-20) reduce to

$$
\begin{array}{rlrl}
\psi_{F_{t}^{d}}-\operatorname{div} \psi_{\nabla F_{t}^{d}} & =T_{R t} & & \text { in } \Omega_{R}, \\
\psi_{\nabla F_{t}^{d}} n_{R}=0 & & \text { on } \partial \Omega_{R} .
\end{array}
$$

Together with (3-18) and (4-18) $)_{1},(5-1)_{1}$ forms the differential system

$$
\operatorname{div} T_{R}+b_{R}=0, \quad \varphi_{F}-\operatorname{div} \varphi_{\nabla F}=T_{R}+B_{R}, \quad \psi_{F^{d}}-\operatorname{div} \psi_{\nabla F^{d}}=T_{R},
$$

in which only the current values of the variables at a fixed instant are involved. Therefore, equations relative to different $t$ are independent. ${ }^{61}$ By consequence, the deformation process $t \mapsto\left(f_{t}, F_{t}\right)$ due to given external actions $t \mapsto\left(b_{R t}, B_{R t}\right)$

${ }^{61}$ For this reason, the subscript $t$ has been omitted. 
and to given boundary data and initial conditions is obtained solving a family of boundary value problems, one for each $t$. No initial conditions are required, and the boundary conditions can be either boundary conditions of place

$$
f=\hat{f}, \quad F=\hat{F}, \quad F^{d}=0,
$$

or boundary conditions of traction

$$
T_{R} n_{R}=\hat{s}_{R}, \quad \varphi_{\nabla F} n_{R}=\hat{S}_{R}, \quad \psi_{\nabla F^{d}} n_{R}=0 .
$$

Equations (5-2)-(5-4) form the equilibrium problem which determines the deformation $(f, F)$ as a function of the external actions $\left(b_{R}, B_{R}\right)$ and of the boundary data $(\hat{f}, \hat{F})$ or $\left(\hat{s}_{R}, \hat{S}_{R}\right)$.

To investigate the well-posedness of this problem, it may be helpful to consider the elementary example of a one-dimensional body

$$
\Omega_{R}=\left\{x_{R}=x_{0}+\lambda\left(x_{l}-x_{0}\right) \mid \lambda \in(0,1)\right\},
$$

subject to null external actions, $b_{R}=B_{R}=0$, and with the quadratic energies

$$
\varphi(F)=\frac{1}{2} k|F|^{2}+\frac{1}{2} K|\nabla F|^{2}, \quad \psi\left(F^{d}\right)=\frac{1}{2} k_{d}\left|F^{d}\right|^{2}+\frac{1}{2} K_{d}\left|\nabla F^{d}\right|^{2},
$$

with $k, K$ and $k_{d}, K_{d}$ given positive constants. Since in one dimension both gradient and divergence coincide with the ordinary derivative, (5-2) reduces to

$$
\nabla T_{R}+b_{R}=0, \quad k F-K \nabla^{2} F=T_{R}+B_{R}, \quad k_{d} F^{d}-K_{d} \nabla^{2} F^{d}=T_{R},
$$

where $\nabla$ now denotes the ordinary derivative. From (5-7) 1 integrated over $\left(x_{0}, x_{R}\right)$ we have the equation

$$
T_{R}\left(x_{R}\right)=T_{R}\left(x_{0}\right)-\int_{x_{0}}^{x_{R}} b_{R}\left(\xi_{R}\right) d \xi_{R},
$$

which determines $T_{R}$ to within the constant $T_{R}\left(x_{0}\right)$. The two remaining equations (5-7) are second-order differential equations with constant coefficients. They admit the closed-form solutions

$$
\begin{array}{rlrl}
F\left(x_{R}\right) & =A \exp \left(\kappa^{2} x_{R}\right)+B \exp \left(-\kappa^{2} x_{R}\right)+\bar{F}\left(x_{R}\right), & & \kappa^{2}=k / K, \\
F^{d}\left(x_{R}\right) & =A^{d} \exp \left(\kappa_{d}^{2} x_{R}\right)+B^{d} \exp \left(-\kappa_{d}^{2} x_{R}\right)+\bar{F}^{d}\left(x_{R}\right), & \kappa_{d}^{2}=k_{d} / K_{d},
\end{array}
$$

where $\bar{F}$ and $\bar{F}^{d}$ are particular solutions of (5-7) $)_{1}$ and (5-7) 2 , respectively.

For example, if $b_{R}$ and $B_{R}$ are the constant fields $\hat{b}_{R}$ and $\hat{B}_{R}$, for $x_{0}=0$ and $x_{l}-x_{0}=l$, we have

$$
k \bar{F}\left(x_{R}\right)=T_{R}(0)+\hat{B}_{R}+\hat{b}_{R} x_{R}, \quad k_{d} \bar{F}^{d}\left(x_{R}\right)=T_{R}(0)+\hat{b}_{R} x_{R} .
$$


The constants $A, B, A^{d}, B^{d}$, and $T_{R}(0)$ are determined by the boundary conditions (5-3) and (5-4). For example, for the boundary conditions of place

$$
f(0)=\hat{f}_{0}, \quad f(l)=\hat{f}_{l}, \quad F(0)=\hat{F}_{0}, \quad F(l)=\hat{F}_{l}, \quad F^{d}(0)=F^{d}(l)=0,
$$

the five constants are the solution of the linear system

$$
\begin{aligned}
& A+B+k^{-1}\left(T_{R}(0)+\hat{B}_{R}\right)=\hat{F}_{0}, \\
& A \exp \left(\kappa^{2} l\right)+B \exp \left(-\kappa^{2} l\right)+k^{-1}\left(T_{R}(0)+\hat{B}_{R}+\hat{b}_{R} l\right)=\hat{F}_{l}, \\
& A^{d}+B^{d}+k_{d}^{-1} T_{R}(0)=0, \\
& A^{d} \exp \left(\kappa_{d}^{2} l\right)+B^{d} \exp \left(-\kappa_{d}^{2} l\right)+k_{d}^{-1}\left(T_{R}(0)+\hat{b}_{R} l\right)=0, \\
& A \kappa^{-2}\left(\exp \left(\kappa^{2} l\right)-1\right)-B \kappa^{-2}\left(\exp \left(-\kappa^{2} l\right)-1\right) \\
&+A^{d} \kappa_{d}^{-2}\left(\exp \left(\kappa_{d}^{2} l\right)-1\right)-B^{d} \kappa_{d}^{-2}\left(\exp \left(-\kappa_{d}^{2} l\right)-1\right) \\
&+k^{-1}\left(\left(T_{R}(0)+\hat{B}_{R}\right) l+\hat{b}_{R} l^{2} / 2\right)+k_{d}^{-1}\left(T_{R}(0) l+\hat{b}_{R} l^{2} / 2\right)=\hat{f}_{l}-\hat{f}_{0},
\end{aligned}
$$

in which the last equation comes from the integration over $(0, l)$ of the relation $F+F^{d}=\nabla f$.

5.2. The rate-dependent dissipative problem. Consider a dissipation potential of the form (4-27), positively homogeneous of order two:

$$
\begin{gathered}
\chi\left(\dot{F}^{d}, \nabla \dot{F}^{d}\right)=\frac{1}{2} \mathbb{M}\left[\dot{F}^{d}\right] \cdot \dot{F}^{d}+\frac{1}{2} \mathbb{K}\left[\nabla \dot{F}^{d}\right] \cdot \nabla \dot{F}^{d}, \\
\mathbb{W}=\chi_{\dot{F}^{d} \dot{F}^{d}}^{\prime}(0), \quad \mathbb{K}=\chi_{\nabla \dot{F}^{d} \nabla \dot{F}^{d}}^{\prime \prime}(0) .
\end{gathered}
$$

Because it describes the dissipation exhibited by viscous materials, this $\chi$ can be called a dissipation potential of the viscous type, and since in the power $\chi\left(\dot{F}^{d}, \nabla \dot{F}^{d}\right)$ the internal actions $\mathbb{U}\left[\dot{F}^{d}\right], \mathbb{K}\left[\nabla \dot{F}^{d}\right]$ depend on the time derivative of $F^{d}$, this $\chi$ is rate-dependent. For $\delta \chi$, from (4-28) we have

$$
\delta \chi\left(\dot{F}^{d}, \nabla \dot{F}^{d}, V^{d}, \nabla V^{d}\right)=\nVdash\left[\dot{F}^{d}\right] \cdot V^{d}+\mathbb{K}\left[\nabla \dot{F}^{d}\right] \cdot \nabla V^{d},
$$

and if $V^{d}$ is allowed to be any second-order tensor field, from (4-19) we get

$$
\begin{aligned}
T_{R t}-\psi_{F_{t}^{d}}+\operatorname{div} \psi_{\nabla F_{t}^{d}}-\mathbb{M}\left[\dot{F}_{t}^{d}\right]+\operatorname{div}\left(\mathbb{K}\left[\nabla \dot{F}_{t}^{d}\right]\right)=0 & \text { in } \Omega_{R}, \\
\left(\psi_{\nabla F_{t}^{d}}+\mathbb{K}\left[\nabla \dot{F}_{t}^{d}\right]\right) n_{R}=0 & \text { on } \partial \Omega_{R} .
\end{aligned}
$$

Due to the presence of $\dot{F}^{d}$ and $\nabla \dot{F}^{d}$, there is a relation between equations written at different times. The family of equilibrium problems (5-2)-(5-4) then merges into a single initial/boundary-value problem, consisting of (3-18), (4-18) $1,(5-15)_{1}$, plus initial conditions $\left(\hat{f}_{0}, \hat{F}_{0}\right)$ over $\Omega_{R}$ and a family $t \mapsto\left(\hat{f}_{t}, \hat{F}_{t}\right)$ or $t \mapsto\left(\hat{s}_{R t}, \hat{S}_{R t}\right)$ of boundary conditions. For this problem, a standard solution strategy is to transform it into a family of incremental problems, one for each $t$, in which the deformation 
( $\left.f_{t}, F_{t}\right)$ and the stress $T_{R t}$, both compatible with (3-18) and (4-18) 1 , are supposed to be known at some instant $t$, and (5-15) is used to determine the derivative $\dot{F}_{t}^{d}$.

For example, in the case of $\varphi, \psi, \chi$ independent of the gradients $\nabla F, \nabla F^{d}, \nabla \dot{F}^{d}$, the tensor $\mathbb{K}$ is zero, $\dot{F}_{t}^{d}$ is determined by the flow rule ${ }^{62}$

$$
\dot{F}_{t}^{d}=\mathbb{H}^{-1}\left[T_{R t}-\psi_{F^{d}}\left(F_{t}^{d}\right)\right],
$$

and $\left(\dot{f}_{t}, \dot{F}_{t}\right)$ and $\dot{T}_{R t}$ are determined solving the incremental problem formed by the incremental versions of (3-18) and (4-18) 1 ,

$$
\operatorname{div} \dot{T}_{R t}+\dot{b}_{R t}=0, \quad \dot{T}_{R t}=\varphi_{F F}\left(F_{t}\right)\left[\dot{F}_{t}\right]-\dot{B}_{R t},
$$

and by the corresponding boundary conditions.

An even more elementary example is the following. Assume that the body is a set of three points, with quadratic energies independent of the gradient,

$$
\varphi(F)=\frac{1}{2} c_{1}|F|^{2}, \quad \psi\left(F^{d}\right)=\frac{1}{2} c_{2}\left|F^{d}\right|^{2}, \quad \chi\left(\dot{F}^{d}\right)=\frac{1}{2} c_{3}\left|\dot{F}^{d}\right|^{2},
$$

and that $b_{R t}=B_{R t}=0$. Then from (4-18) 1 and (5-15) 1 we have

$$
T_{R t}=c_{1} F_{t}, \quad T_{R t}=c_{2} F_{t}^{d}+c_{3} \dot{F}_{t}^{d} .
$$

These are the equations of the Kelvin-Voigt model, which consists of a spring of stiffness $c_{1}$, in series with a system made of a second spring of stiffness $c_{2}$ and a piston of stiffness $c_{3}$, set in parallel. The elongation of the first spring is $F_{t}$, and $F_{t}^{d}$ is the elongation of both the second spring and the piston. The corresponding internal actions are $c_{1} F_{t}, c_{2} F_{t}^{d}, c_{3} \dot{F}_{t}^{d}$, the external action is $T_{R t}$, and (5-19) are the balance equations between internal and external actions. By elimination of $T_{R t}$, we get the differential equation

$$
c_{3} \dot{F}_{t}^{d}+c_{2} F_{t}^{d}=c_{1} F_{t} .
$$

The boundary condition of place consists of prescribing the total elongation

$$
\hat{F}_{t}^{\text {tot }}=F_{t}+F_{t}^{d}
$$

at all $t$, and the initial conditions consist of prescribing $F_{t}$ and $F_{t}^{d}$ at $t=0$. However, due to the compatibility condition $F_{0}+F_{0}^{d}=\hat{F}_{0}^{\text {tot }}$ at $t=0$, only one of the conditions $F_{0}=\hat{F}_{0}$ or $F_{0}^{d}=\hat{F}_{0}^{d}$ is required. Thus, we have the differential problem

$$
\dot{F}_{t}^{d}+k F_{t}^{d}=c_{1} c_{3}^{-1} \hat{F}_{t}^{\text {tot }}, \quad k=\left(c_{1}+c_{2}\right) c_{3}^{-1},
$$

with $\hat{F}_{t}^{\text {tot }}$ given and with, for example, $F_{0}=\hat{F}_{0}$. The solution is

$$
F_{t}^{d}=A \exp (-k t)+\bar{F}_{t},
$$

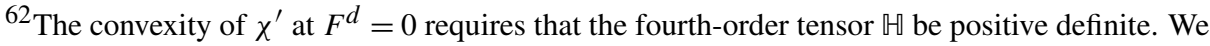
assume that it is in fact positive definite. 
where $\bar{F}_{t}$ is a solution of the nonhomogeneous equation (5-22), and the constant $A$ is determined by the initial condition

$$
\hat{F}_{0}^{\text {tot }}=\hat{F}_{0}+A+\bar{F}_{0}
$$

which is $(5-21)$ at $t=0$.

5.3. The rate-independent dissipative problem. For a dissipation potential positively homogeneous of order one of the form (4-27), from (4-23) we have

$$
\chi\left(\dot{F}^{d}, \nabla \dot{F}^{d}\right)=\chi_{\dot{F}^{d}}^{\prime}(0) \triangleright \dot{F}^{d}+\chi_{\nabla \dot{F}^{d}}^{\prime \prime}(0) \triangleright \nabla \dot{F}^{d} .
$$

Since it describes the dissipation exhibited by plastic materials, this $\chi$ can be called a dissipation potential of the plastic type, and because in the power $\chi\left(\dot{F}^{d}, \nabla \dot{F}^{d}\right)$ the internal actions $\chi_{\dot{F}^{d}}^{\prime}(0), \chi_{\nabla \dot{F}^{d}}^{\prime \prime}(0)$ do not depend on time derivatives, this $\chi$ is rateindependent. Then from (4-19) and from the upper bound (4-29) on $\delta \chi$ we have

$$
\begin{aligned}
\int_{\Omega_{R}}\left(\left(T_{R t}-\psi_{F_{t}^{d}}+\operatorname{div} \psi_{\nabla F_{t}^{d}}\right) \cdot V^{d}-\breve{\chi}_{\dot{F}^{d}}^{\prime}(0) \triangleright V^{d}-\breve{\chi}_{\nabla \dot{F}^{d}}^{\prime \prime}(0) \triangleright \nabla V^{d}\right) \mathrm{d} v & \\
& -\int_{\partial \Omega_{R}} \psi_{\nabla F_{t}^{d}} \cdot\left(V^{d} \otimes n_{R}\right) \mathrm{da} \leq 0,
\end{aligned}
$$

and from the divergence formula (A-4) in the Appendix we get

$$
\begin{aligned}
\int_{\Omega_{R}}\left(\left(T_{R t}-\psi_{F_{t}^{d}}\right.\right. & \left.\left.+\operatorname{div} \psi_{\nabla F_{t}^{d}}\right) \cdot V^{d}-\left(\breve{\chi}_{\dot{F}_{t}^{d}}^{\prime}(0)-\operatorname{div} \breve{\chi}_{\nabla \dot{F}_{t}^{d}}^{\prime \prime}(0)\right) \triangleright V^{d}\right) \mathrm{dv} \\
& -\int_{\partial \Omega_{R}}\left(\psi_{\nabla F_{t}^{d}} \cdot\left(V^{d} \otimes n_{R}\right)+\breve{\chi}_{\nabla \dot{F}^{d}}^{\prime \prime}(0) \triangleright\left(V^{d} \otimes n_{R}\right)\right) \mathrm{da} \leq 0 .
\end{aligned}
$$

For $V^{d}=\dot{F}_{t}^{d}$ the inequality turns to equality, and the punctual conditions

$$
\begin{aligned}
\left(T_{R t}-\psi_{F_{t}^{d}}+\operatorname{div} \psi_{\nabla F_{t}^{d}}\right) \cdot \dot{F}_{t}^{d}-\left(\breve{\chi}_{\dot{F}_{t}^{d}}^{\prime}(0)-\operatorname{div} \breve{\chi}_{\nabla \dot{F}_{t}^{d}}^{\prime \prime}(0)\right) \triangleright \dot{F}_{t}^{d}=0 & \text { in } \Omega_{R}, \\
\psi_{\nabla F_{t}^{d}} n_{R} \cdot \dot{F}_{t}^{d}+\breve{\chi}_{\nabla \dot{F}_{t}^{d}}^{\prime \prime}(0) \triangleright\left(\dot{F}_{t}^{d} \otimes n_{R}\right)=0 & \text { on } \partial \Omega_{R}
\end{aligned}
$$

follow. Calling $N_{t}$ the direction $\dot{F}_{t}^{d} /\left|\dot{F}_{t}^{d}\right|$ of $\dot{F}_{t}^{d}$, the first equation can be given the form

$$
\left|\dot{F}_{t}^{d}\right|\left(\left(T_{R t}-\psi_{F_{t}^{d}}+\operatorname{div} \psi_{\nabla F_{t}^{d}}\right) \cdot N_{t}-\left(\breve{\chi}_{\dot{F}_{t}^{d}}^{\prime}(0)-\operatorname{div} \breve{\chi}_{\nabla \dot{F}_{t}^{d}}^{\prime \prime}(0)\right) \triangleright N_{t}\right)=0 .
$$

It tells us that a nonnull deformation $\dot{F}_{t}^{d}$ can occur only if there is a direction $N_{t}$ for which the term between parentheses is zero. This is the activation condition for the strain rate $\dot{F}_{t}^{d}$, and

$$
\dot{F}_{t}^{d}= \begin{cases}\left|\dot{F}_{t}^{d}\right| N_{t} & \text { if } N_{t} \text { is a solution of problem (5-28), } \\ 0 & \text { otherwise }\end{cases}
$$


is the corresponding flow rule. The determination of $N_{t}$ requires the solution of the differential problem (5-28). Once again we have an initial/boundary-value problem, which can be reduced to a family of incremental problems. Each problem is formulated like in the rate-dependent case, with the nonlocal flow rule (5-30) in place of the local flow rule $(5-16) .{ }^{63}$

For $\chi^{\prime \prime}=0$ and $\psi$ independent of $\nabla F^{d}$, which is the case of classical plasticity, the local form of inequality (5-27) is

$$
\left(T_{R t}-\psi_{F^{d}}\left(F_{t}^{d}\right)\right) \cdot V^{d}-\breve{\chi}_{\dot{F}^{d}}(0) \triangleright V^{d} \leq 0,
$$

(5-29) reduces to

$$
\left|\dot{F}_{t}^{d}\right|\left(\left(T_{R t}-\psi_{F^{d}}\left(F_{t}^{d}\right)\right) \cdot N_{t}-\breve{\chi}_{\dot{F}^{d}}(0) \triangleright N_{t}\right)=0,
$$

the flow rule (5-30) reduces to

$$
\dot{F}_{t}^{d}= \begin{cases}\left|\dot{F}_{t}^{d}\right| N_{t} & \text { if }\left(T_{R t}-\psi_{F^{d}}\left(F_{t}^{d}\right)\right) N_{t}=\breve{\chi}_{\dot{F}^{d}}(0) \triangleright N_{t}, \\ 0 & \text { if }\left(T_{R t}-\psi_{F^{d}}\left(F_{t}^{d}\right)\right) N_{t}<\breve{\chi}_{\dot{F}^{d}}(0) \triangleright N_{t},\end{cases}
$$

and the differential problem (5-28) reduces to the determination of a unit tensor $N_{t}$ for which the term between parentheses in (5-32) is zero. ${ }^{64}$ The initial/boundaryvalue problem can still be reduced to a family of incremental problems, whose field equations are the incremental equations (5-17) and the flow rule (5-33).

5.4. A comparison with Gurtin and Anand's theory. It is instructive to compare the present theory with the theory of gradient plasticity of Gurtin and Anand. ${ }^{65}$ The two theories differ in the forms assumed for the equation of virtual power. The form assumed by Gurtin and Anand is ${ }^{66}$

$$
\int_{\Omega_{R}} b_{R} \cdot v \mathrm{dv}+\int_{\partial \Omega_{R}}\left(s_{R} \cdot v+S_{R} \cdot V^{d}\right) \mathrm{da}=\int_{\Omega_{R}}\left(T_{R} \cdot V+T_{R}^{d} \cdot V^{d}+\mathbb{T}_{R} \cdot \nabla V^{d}\right) \mathrm{d} v .
$$

${ }^{63}$ Note that if $\dot{F}_{t}^{d}$ is a solution to problem (5-28), then $\lambda \dot{F}_{t}^{d}$ is a solution for all $\lambda \geq 0$. Correspondingly, the flow rule (5-30) specifies the direction, but not the amplitude, of the strain rate $\dot{F}_{t}^{d}$.

${ }^{64}$ In the paper [Del Piero 2018c] it has been shown that the set of all $T_{R t}$ for which this parenthesis is nonpositive for all $V^{d}$ is a convex set in the space of the second-order tensors. This set is the elastic range, and the tensor $\psi_{F^{d}}$ is the backstress tensor $T_{B t}$ of kinematic plasticity. A plastic deformation can be activated only if the tensor $\left(T_{R t}-T_{B t}\right)$ is located on the yield surface, which is the boundary of the elastic range. In this case, $N_{t}$ belongs to the normal cone to the yield surface at $\left(T_{R t}-T_{B t}\right)$. This is the normality law (5-33). As pointed out in [Del Piero 2018c], what distinguishes the present approach from the traditional ones is that the activation condition, elastic range, backstress tensor, and normality law are not assumed a priori, but systematically deduced as consequences of a specific choice of the dissipation potential.

${ }^{65}$ See [Gurtin and Anand 2005]. Here and in the following I make reference to the version given in [Gurtin et al. 2010, §90]. To make the comparison easier, wherever possible I translated the notations of [Gurtin et al. 2010] into the ones of the present paper.

${ }^{66}$ See [Gurtin et al. 2010, (90.16)]. This form of the principle of virtual power was proposed by Fleck and Hutchinson [1993] with a scalar measure of plastic strain, and extended later to a tensorial measure by Gudmundson [2004]. See also [Fleck and Willis 2009a; 2009b]. 
Comparing with (4-16) above, we see that in the external power the action $B_{R}$ is missing, and that the velocity associated with the contact action $S_{R}$ is $V^{d}$ instead of $V$. In the internal power, the internal actions are the stress tensors $T_{R}, T_{R}^{d}, \mathbb{T}_{R}$. No motivations are given for neglecting $B_{R}$, for taking $V^{d}$ as independent virtual velocity, and for introducing the stress tensors $T_{R}^{d}$ and $\mathbb{T}_{R}$.

With the aid of the divergence theorem and of the relation $V=\nabla v-V^{d}$, (5-34) takes the form

$$
\begin{aligned}
\int_{\Omega_{R}}\left(\left(\operatorname{div} T_{R}+b_{R}\right) \cdot v\right. & \left.+\left(T_{R}-T_{R}^{d}+\operatorname{div} \mathbb{T}_{R}\right) \cdot V^{d}\right) \mathrm{d} v \\
& +\int_{\partial \Omega_{R}}\left(\left(s_{R}-T_{R} n_{R}\right) \cdot v+\left(S_{R}-\mathbb{T}_{R} n_{R}\right) \cdot V^{d}\right) \mathrm{da}=0,
\end{aligned}
$$

and from the arbitrariness of $v$ and $V^{d}$ the local equations ${ }^{67}$

$$
\begin{aligned}
& \operatorname{div} T_{R}+b_{R}=0, \quad T_{R}-T_{R}^{d}+\operatorname{div} \mathbb{T}_{R}=0 \quad \text { in } \Omega_{R}, \\
& s_{R}=T_{R} n_{R}, \quad S_{R}=\mathbb{T}_{R} n_{R} \quad \text { in } \partial \Omega_{R}
\end{aligned}
$$

follow. Moreover, $T_{R}^{d}$ and $\mathbb{T}_{R}$ are split into the sum of an energetic and a dissipative part. For the energetic parts, a comparison between (5-36) 1 and (4-19) suggests the identifications

$$
T_{R / \mathrm{en}}^{d}=\psi_{F^{d}}\left(F^{d}, \nabla F^{d}\right), \quad \mathbb{T}_{R / \mathrm{en}}=\psi_{\nabla F^{d}}\left(F^{d}, \nabla F^{d}\right) .
$$

As for the dissipative parts, from (4-19) we have

$$
T_{R / \text { dis }}^{d} \cdot \dot{F}^{d}+\mathbb{T}_{R / \text { dis }} \cdot \nabla \dot{F}^{d}=-\delta \chi\left(\dot{F}^{d}, \nabla \dot{F}^{d}, \dot{F}^{d}, \nabla \dot{F}^{d}\right),
$$

and from (4-28) and (4-29) it follows that

$$
T_{R / \mathrm{dis}}^{d}=\chi_{\dot{F}^{d} \dot{F}^{d}}^{\prime}(0)\left[\dot{F}^{d}\right], \quad \mathbb{T}_{R / \mathrm{dis}}=\chi_{\nabla^{d} \dot{F}^{d}}^{\prime \prime}(0)\left[\nabla \dot{F}^{d}\right]
$$

for rate-dependent dissipation potentials, and

$$
T_{R / \text { dis }}^{d}=\breve{\chi}_{\dot{F}^{d}}^{\prime}(0), \quad \mathbb{T}_{R / \text { dis }}=\breve{\chi}_{\nabla \dot{F}^{d}}^{\prime \prime}(0)
$$

for rate-independent dissipation potentials. ${ }^{68}$

In [Gurtin et al. 2010], (5-36) 1,2 are the macroscopic and microscopic force balances, while (5-37), (5-39), and (5-40) are constitutive equations relating the

${ }^{67}$ See equations (90.18), (90.23), (90.17), and (90.24) of [Gurtin et al. 2010], respectively.

${ }^{68}$ In [Gurtin et al. 2010] the stress $T_{R}^{d}$ is assumed to be totally dissipative, with constitutive equation (90.54). For $\mathbb{T}_{R}$ the constitutive equations for the energetic and dissipative parts are (90.47) and (90.59) 2 , respectively. For $\mathbb{T}_{R / \text { dis }}$ the identifications (5-39) 2 and (5-40) 2 are only approximative, because in [Gurtin et al. 2010] all constitutive functions are differentiable and there are no dissipation potentials. 
forces $T_{R}, T_{R}^{d}, \mathbb{T}_{R}$ with the deformations $F, F^{d}$, and the flow rules (5-16) and (5-30) roughly correspond to (90.65) of [Gurtin et al. 2010]. ${ }^{69}$

In the present theory there is only one force, the Piola stress $T_{R}$, and only one constitutive equation, given by $(4-18)_{1}$ :

$$
T_{R}+B_{R}=\varphi_{F}-\operatorname{div} \varphi_{\nabla F} .
$$

The stress tensors $T_{R}^{d}$ and $\mathbb{T}_{R}$ are artificial entities, not really required by the theory. Indeed, in the reduced equation of virtual power (4-19) the internal actions are the partial derivatives of $\varphi, \psi, \chi$ specified in the right-hand sides of (5-37)-(5-39). These derivatives contain all available information about the nature of the material response.

Since the internal actions are already expressed in terms of deformation, there is no need to invent stress tensors and constitutive equations relating them to the deformation. On the contrary, such a redundant procedure could be misleading because, while a constitutive equation is restricted only by indifference conditions, the internal actions may be interrelated by the fact of being partial derivatives of the same energy densities.

\section{Appendix: The divergence of gradient-dependent positively homogeneous functions of order one}

Let $\chi=\chi\left(\nabla \dot{F}^{d}\right)$ be a function with the same properties as the function $\chi^{\prime \prime}$ in Section 5.3: convex, differentiable everywhere except at $\nabla \dot{F}^{d}=0$, and positively homogeneous of order one. For such functions the directional derivatives in the direction $\nabla V^{d}$ are the same at zero and at $\nabla V^{d}$ :

$$
\breve{\chi}_{\nabla \dot{F}^{d}}(0) \triangleright \nabla V^{d}=\chi_{\nabla \dot{F}^{d}}\left(\nabla V^{d}\right) \cdot \nabla V^{d} .
$$

Then, by the divergence theorem,

$$
\begin{aligned}
\int_{\Omega_{R}} \breve{\chi}_{\nabla \dot{F}^{d}}(0) & \triangleright \nabla V^{d} \mathrm{dv}=\int_{\Omega_{R}} \chi_{\nabla \dot{F}^{d}}\left(\nabla V^{d}\right) \cdot \nabla V^{d} \mathrm{dv} \\
= & -\int_{\Omega_{R}} \operatorname{div} \chi_{\nabla \dot{F}^{d}}\left(\nabla V^{d}\right) \cdot V^{d} \mathrm{dv}+\int_{\partial \Omega_{R}} \chi_{\nabla \dot{F}^{d}}\left(\nabla V^{d}\right) n_{R} \cdot V^{d} \mathrm{da} .
\end{aligned}
$$

${ }^{69}$ Almost all current theories of generalized continua include one or more equations of microscopic force balance [Capriz 1989; Germain 1973b; Fleck and Hutchinson 1993; Gurtin et al. 2010]. The nature of such equations is far from being clear. It was just believed that the microscopic forces should be somehow balanced. In the more fundamental approaches [Gurtin and Martins 1976; Noll 1973; Šilhavý 2008], the macroscopic balance of linear momentum (3-18) was deduced from a boundedness assumption on the system of contact actions. In the microscopic scale, this was the approach followed in my previous papers [Del Piero 2009; 2014a; 2014b; 2017; 2018a; 2019], in which equations of the form (5-36) 2 were called pseudobalance equations because they were not expressing the balance of any physical quantity. Here, (5-36) 2 is a direct consequence of energy conservation. Therefore, it does not need any further physical characterization. 
The divergence and boundary values of $\chi_{\nabla \dot{F}^{d}}$ can be extended to the singular point $\nabla V^{d}=0$ by defining

$$
\begin{gathered}
\left(\operatorname{div} \breve{\chi}_{\nabla \dot{F}^{d}}(0)\right) \triangleright V^{d}=\operatorname{div} \chi_{\nabla \dot{F}^{d}}\left(\nabla V^{d}\right) \cdot V^{d}, \\
\breve{\chi}_{\nabla F^{d}}(0) \triangleright\left(V^{d} \otimes n_{R}\right)=\chi_{\nabla \dot{F}^{d}}\left(\nabla V^{d}\right) \cdot\left(V^{d} \otimes n_{R}\right) .
\end{gathered}
$$

Then at $\nabla V^{d}=0$ we have

$$
\int_{\Omega_{R}}\left(\breve{\chi}_{\nabla \dot{F}^{d}}(0) \triangleright \nabla V^{d}+\left(\operatorname{div} \breve{\chi}_{\nabla \dot{F}^{d}}\right)(0) \triangleright V^{d}\right) \mathrm{dv}=\int_{\partial \Omega_{R}} \breve{\chi}_{\nabla \dot{F}^{d}}(0) \triangleright\left(V^{d} \otimes n_{R}\right) \mathrm{da} .
$$

This can be regarded as a generalized version of the divergence theorem at the singular point $\nabla V^{d}=0$.

\section{References}

[Aifantis 1984] E. C. Aifantis, "On the microstructural origin of certain inelastic models", ASME J. Eng. Mater. Technol. 106:4 (1984), 326-330.

[Barroso et al. 2017] A. C. Barroso, J. Matias, M. Morandotti, and D. R. Owen, "Second-order structured deformations: relaxation, integral representation and applications", Arch. Ration. Mech. Anal. 225:3 (2017), 1025-1072.

[Capriz 1989] G. Capriz, Continua with microstructure, Springer Tracts in Natural Philosophy 35, Springer, 1989.

[Choksi and Fonseca 1997] R. Choksi and I. Fonseca, "Bulk and interfacial energy densities for structured deformations of continua”, Arch. Rational Mech. Anal. 138:1 (1997), 37-103.

[Coleman and Noll 1963] B. D. Coleman and W. Noll, "The thermodynamics of elastic materials with heat conduction and viscosity", Arch. Rational Mech. Anal. 13 (1963), 167-178.

[Del Piero 2001] G. Del Piero, "The energy of a one-dimensional structured deformation”, Math. Mech. Solids 6:4 (2001), 387-408.

[Del Piero 2009] G. Del Piero, "On the method of virtual power in continuum mechanics", J. Mech. Mater. Struct. 4:2 (2009), 281-292.

[Del Piero 2014a] G. Del Piero, "Nonclassical continua, pseudobalance, and the law of action and reaction", Math. Mech. Complex Syst. 2:1 (2014), 71-107.

[Del Piero 2014b] G. Del Piero, "On the method of virtual power in the mechanics of non-classical continua", pp. 29-58 in Multiscale modeling of complex materials: phenomenological, theoretical and computational aspects, edited by T. Sadowski and P. Trovalusci, CISM Courses and Lectures 556, Springer, 2014.

[Del Piero 2017] G. Del Piero, "Une approche rationnelle des milieux continus avec microstructure", pp. 11-114 in Mécanique des milieux continus généralisés, Cépaduès, Toulouse, France, 2017.

[Del Piero 2018a] G. Del Piero, "An axiomatic framework for the mechanics of generalized continua”, Atti Accad. Naz. Lincei Rend. Lincei Mat. Appl. 29:1 (2018), 31-61.

[Del Piero 2018b] G. Del Piero, "On the decomposition of the deformation gradient in plasticity", $J$. Elasticity 131:1 (2018), 111-124.

[Del Piero 2018c] G. Del Piero, "The variational structure of classical plasticity", Math. Mech. Complex Syst. 6:3 (2018), 137-180. 
[Del Piero 2019] G. Del Piero, “'Reality' and representation in mechanics: the legacy of Walter Noll”, J. Elasticity 135:1-2 (2019), 117-148.

[Del Piero 2020] G. Del Piero, "A mechanical model for heat conduction”, Contin. Mech. Thermodyn. 32:4 (2020), 1159-1172.

[Del Piero and Owen 1993] G. Del Piero and D. R. Owen, "Structured deformations of continua", Arch. Rational Mech. Anal. 124:2 (1993), 99-155.

[Del Piero and Owen 1995] G. Del Piero and D. R. Owen, "Integral-gradient formulae for structured deformations", Arch. Rational Mech. Anal. 131:2 (1995), 121-138.

[Fleck and Hutchinson 1993] N. A. Fleck and J. W. Hutchinson, "A phenomenological theory for strain gradient effects in plasticity”, J. Mech. Phys. Solids 41:12 (1993), 1825-1857.

[Fleck and Willis 2009a] N. A. Fleck and J. R. Willis, "A mathematical basis for strain-gradient plasticity theory, I: Scalar plastic multiplier”, J. Mech. Phys. Solids 57:1 (2009), 161-177.

[Fleck and Willis 2009b] N. A. Fleck and J. R. Willis, "A mathematical basis for strain-gradient plasticity theory, II: Tensorial plastic multiplier”, J. Mech. Phys. Solids 57:7 (2009), 1045-1057.

[Germain 1973a] P. Germain, "La méthode des puissances virtuelles en mécanique des milieux continus, I: Théorie du second gradient", J. Mécanique 12 (1973), 235-274.

[Germain 1973b] P. Germain, "The method of virtual power in continuum mechanics, 2: Microstructure", SIAM J. Appl. Math. 25:3 (1973), 556-575.

[Germain et al. 1983] P. Germain, Q. S. Nguyen, and P. Suquet, "Continuum thermodynamics", ASME J. Appl. Mech. 50:4b (1983), 1010-1020.

[Green and Rivlin 1957] A. E. Green and R. S. Rivlin, "The mechanics of non-linear materials with memory", Arch. Rational Mech. Anal. 1 (1957), 1-21.

[Gudmundson 2004] P. Gudmundson, "A unified treatment of strain gradient plasticity", J. Mech. Phys. Solids 52:6 (2004), 1379-1406.

[Gurtin 1981] M. E. Gurtin, An introduction to continuum mechanics, Mathematics in Science and Engineering 158, Academic Press, New York, 1981.

[Gurtin and Anand 2005] M. E. Gurtin and L. Anand, "A theory of strain-gradient plasticity for isotropic, plastically irrotational materials, II: Finite deformations”, Int J. Plasticity 21:12 (2005), 2297-2318.

[Gurtin and Martins 1976] M. E. Gurtin and L. C. Martins, "Cauchy's theorem in classical physics", Arch. Rational Mech. Anal. 60:4 (1976), 305-324.

[Gurtin and Murdoch 1975] M. E. Gurtin and A. I. Murdoch, "A continuum theory of elastic material surfaces”, Arch. Rational Mech. Anal. 57 (1975), 291-323.

[Gurtin et al. 2010] M. E. Gurtin, E. Fried, and L. Anand, The mechanics and thermodynamics of continua, Cambridge University Press, 2010.

[Hackl and Fischer 2008] K. Hackl and F. D. Fischer, "On the relation between the principle of maximum dissipation and inelastic evolution given by dissipation potentials", Proc. R. Soc. Lond. Ser. A Math. Phys. Eng. Sci. 464:2089 (2008), 117-132.

[Halphen and Son 1975] B. Halphen and N. Q. Son, "Sur les matériaux standards généralisés", J. Mécanique 14 (1975), 39-63.

[Jammer 1999] M. Jammer, Concepts of force, Dover, Mineola, NY, 1999.

[Mach 1883] E. Mach, Die Mechanik in ihrer Entwickelung: historisch-kritisch dargestellt, Brockhaus, Leipzig, 1883. Translated as The science of mechanics: a critical and historical account of its development, 2nd ed., Open Court, Chicago, 1919. 
[Mach 1896] E. Mach, Die Principien der Wärmelehre, Barth, Leipzig, 1896. Translated as Principles of the theory of heat, 2nd ed., Open Court, Chicago, 1904.

[Moreau 1970] J. J. Moreau, "Sur les lois de frottement, de plasticité et de viscosité", C. R. Acad. Sci. Paris Sér. A 271 (1970), 608-611.

[Moreau 1974] J.-J. Moreau, "On unilateral constraints, friction and plasticity”, pp. 171-322 in New variational techniques in mathematical physics (Bressanone, Italy, 1973), edited by G. Capriz and G. Stampacchia, Cremonese, Rome, 1974.

[Murdoch 2012] A. I. Murdoch, Physical foundations of continuum mechanics, Cambridge University Press, 2012.

[Noll 1959] W. Noll, "The foundations of classical mechanics in the light of recent advances in continuum mechanics", pp. 266-281 in The axiomatic method: with special reference to geometry and physics, edited by L. Henkin et al., North-Holland, Amsterdam, 1959. Reprinted in [Noll 1974, pp. 31-47].

[Noll 1963] W. Noll, "La mécanique classique basée sur un axiome d'objectivité", pp. 47-56 in La méthode axiomatique dans les mécaniques classiques et nouvelles, edited by A. Chatelet, Les Grands Problèmes 13, Gauthier-Villars, Paris, 1963. Reprinted in [Noll 1974, pp. 135-144].

[Noll 1973] W. Noll, "Lectures on the foundations of continuum mechanics and thermodynamics", Arch. Rational Mech. Anal. 52 (1973), 62-92. Reprinted in [Noll 1974, pp. 293-324].

[Noll 1974] W. Noll, The foundations of mechanics and thermodynamics: selected papers, Springer, 1974.

[Noll 1995] W. Noll, “On material frame-indifference”, research report 95-NA-022, Carnegie Mellon University, 1995.

[Noll 2004] W. Noll, "Updating The Non-Linear Field Theories of Mechanics", pp. 23-39 in Five contributions to natural philosophy, 2004.

[Noll 2006] W. Noll, "On the past and future of natural philosophy", J. Elasticity 84:1 (2006), 1-11. [Owen 2000] D. R. Owen, "Structured deformations, II", lecture notes 58, Ravello Summer School of the Gruppo Nazionale di Fisica Matematica, 2000, Available at http://www.math.cmu.edu/users/ nw0z/publications/99-CNA-014/99-CNA-014/.

[Reina and Conti 2014] C. Reina and S. Conti, "Kinematic description of crystal plasticity in the finite kinematic framework: a micromechanical understanding of $\mathbf{F}=\mathbf{F}^{e} \mathbf{F} p "$, J. Mech. Phys. Solids 67 (2014), 40-61.

[Truesdell 1991] C. Truesdell, A first course in rational continuum mechanics, vol. 1: General concepts, 2nd ed., Pure and Applied Mathematics 71, Academic Press, Boston, 1991.

[Truesdell and Noll 1965] C. Truesdell and W. Noll, The non-linear field theories of mechanics, edited by S. Flügge, Handbuch der Physik III-3, Springer, 1965.

[Šilhavý 2008] M. Šilhavý, “Cauchy’s stress theorem for stresses represented by measures”, Contin. Mech. Thermodyn. 20:2 (2008), 75-96.

[Šilhavý 2017] M. Šilhavý, "The general form of the relaxation of a purely interfacial energy for structured deformations”, Math. Mech. Complex Syst. 5:2 (2017), 191-215.

[Ziegler 1963] H. Ziegler, "Some extremum principles in irreversible thermodynamics with application to continuum mechanics", pp. 91-193 in Progress in solid mechanics, vol. IV, edited by I. N. Sneddon and R. Hill, North-Holland, Amsterdam, 1963.

Received 27 Jan 2020. Revised 31 May 2020. Accepted 8 Jul 2020.

GianPIETRo Del Piero: dlpgpt@unife.it

Dipartimento di Ingegneria, Università di Ferrara, Ferrara, Italy
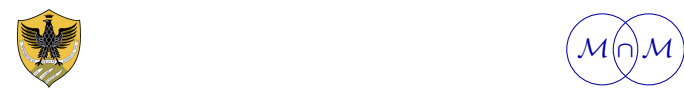